\title{
Window View Access in Architecture: Spatial Visualization and Probability Evaluations Based on Human Vision Fields and Biophilia
}

\author{
Mojtaba Parsaee ${ }^{1, *(1)}$, Claude M. H. Demers ${ }^{1}$, André Potvin ${ }^{1}$, Marc Hébert ${ }^{2}$ and Jean-François Lalonde ${ }^{3}$ \\ 1 GRAP (Groupe de Recherche en Ambiances Physiques), School of Architecture, Laval University, \\ Quebec City, QC G1V 0A6, Canada; Claude.Demers@arc.ulaval.ca (C.M.H.D.); \\ Andre.Potvin@arc.ulaval.ca (A.P.) \\ 2 CERVO Brain Research Centre, Faculty of Medicine, Laval University, Quebec City, QC G1V 0A6, Canada; \\ Marc.Hebert@fmed.ulaval.ca \\ 3 Computer Vision and Systems Lab, Department of Electrical and Computer Engineering, Laval University, \\ Quebec City, QC G1V 0A6, Canada; Jean-Francois.Lalonde@gel.ulaval.ca \\ * Correspondence: Mojtaba.Parsaee.1@ulaval.ca
}

check for updates

Citation: Parsaee, M.; Demers, C.M.H.; Potvin, A.; Hébert, M.; Lalonde, J.-F. Window View Access in Architecture: Spatial Visualization and Probability Evaluations Based on Human Vision Fields and Biophilia. Buildings 2021, 11, 627. https:/ / doi.org/10.3390/buildings11120627

Academic Editor: Isaac

Guedi Capeluto

Received: 18 October 2021

Accepted: 30 November 2021

Published: 8 December 2021

Publisher's Note: MDPI stays neutral with regard to jurisdictional claims in published maps and institutional affiliations.

Copyright: (c) 2021 by the authors. Licensee MDPI, Basel, Switzerland. This article is an open access article distributed under the terms and conditions of the Creative Commons Attribution (CC BY) license (https:// creativecommons.org/licenses/by/ $4.0 /)$.

\begin{abstract}
This paper presents a computational method for spatial visualization and probability evaluations of window view access in architecture based on human eyes' vision fields and biophilic recommendations. Window view access establishes occupants' visual connections to outdoors. Window view access has not, yet, been discussed in terms of the typical vision fields and related visual experiences. Occupants' views of outdoors could change from almost blocked and poor to good, wide, and immersive visions in relation to the binocular focus to monocular (far-) peripheral sights of human eyes. The proposed methodological framework includes spatial visualizations and cumulative distribution functions of window view access based on visual experiences of occupants. The framework is integrated with biophilic recommendations and existing rating systems for view evaluations. As a pilot study, the method is used to evaluate occupants' view access in a space designed with 15 different configurations of windows and overhangs. Results characterize likelihood of experiencing various field of views (FOVs) in case studies. In particular, window-to-wall-area ratios of between $40 \%$ and $70 \%$ offer optimum distributions of view access in space by offering $75 \%$ likelihoods of experiencing good to wide views and less than $25 \%$ probabilities of exposing to poor and almost blocked views. Results show the contribution of the proposed method to informative decision-making processes in architecture.
\end{abstract}

Keywords: indoor-outdoor connections; computer-aided design; parametric building simulation; biophilic architecture

\section{Introduction}

\subsection{View Access to Outdoors in Architecture}

This research focuses on window view access of occupants in architecture as a primary element of visual connections to outdoors. View access is defined as a metric quantifying the amount of a window through which an occupant could view outdoors from a particular spot in the space [1,2]. Outdoor views are essential features of biophilic architecture and healthy buildings. The theory of biophilia articulates the inherent tendency of humans to connect with nature and naturalistic features, particularly outside buildings [3-5] Viewing outdoors through windows could establish occupants' visual connections to natural phenomena outside buildings [3,5-7]. Immersive visual connections to the outdoor nature could provide high biophilic qualities in architecture [3,5-8]. Biophilic recommendations for outdoor views could contribute to occupants' wellbeing, such as (i) increasing positive, physical, emotional, and cognitive responses and decreasing negative emotions [9-13], 
(ii) reducing stress, anxiety, depression, and patients' recovery time, (iii) regulating circadian rhythms and sleep/awake cycles $[4,5,7,14-16]$, and (iv) promoting job satisfactions [17-19]. Furthermore, spaces with high outdoor view access through windows are shown having higher financial values compared to windowless spaces [20]. Standards and guidelines for designing healthy buildings, including the European Committee For Standardization (CEN) [1], WELL [21], LEED [22], International Living Future Institute [23], Heschong Mahone Group [24], recommend providing occupants with acceptable to high view access to outdoors. Biophilic studies and existing guidelines have not yet offered a metric to quantify occupants' visual experiences and immersive views of outdoors and natural elements.

Further methodological developments and informative spatial visualizations are required to comprehend the impact of architectural alternatives on window view access and occupants' visual experiences of outdoors. Occupants' outdoor view is most often studied in relation to existing rating systems, qualities and visibility of the scene, and daylighting conditions. Previous research called to study window view access as an independent quantitative factor prior to evaluating qualities of views, or visibility and clarity of exterior elements, e.g., the sky, ground, and greenery $[2,20]$. As further discussed in the background section, existing methods also require further developments in terms of quantifiable metrics, thresholds, and occupant-centric spatial evaluations [2,20,25]. Metrics and spatial evaluations for view access in space must be developed to show impacts of architectural variables on potential visual experiences of occupants throughout space [25-27]. Thus, having an efficient view to outdoors through windows must be evaluated in relation to the potential visual experience of an observer prior to discussing qualities of views or visibility of exterior elements.

\subsection{General Objective and Structure of the Paper}

This research aims at developing a methodological framework for window view access which enables discussing potentials of architectural configurations in terms of occupants visual experiences and biophilic connections to outdoors. The method is aimed to include a parametric design workflow and informative visualizations of window view access probabilities to enable architects exploring impacts of different design scenarios. Section 2.1 discusses the shortcomings of existing rating systems and recommendations for window view access in relation to the human eyes' anatomy and typical vision fields. Section 2.2 discusses the potentials and limitations of existing modeling approaches to evaluate view access in space. Section 3 presents details of the developed methodological framework for the spatial visualization and probability evaluations of view access based on human eyes' field of views (FOVs). The method is scripted in the Grasshopper environment of Rhinoceros software coupled with Python data visualization libraries. Note that the method can be scripted in platforms as the details are completely presented in the paper. A pilot study is, then, presented aimed at exploring the efficiency of the proposed method in evaluating window view access potentials in different architectural configurations. The pilot study includes 15 configurations of a generic space designed with different sizes and numbers of windows and external overhangs. Section 4 presents outputs of the pilot study in terms of spatial visualization and probability evaluations of occupants' view access in the case studies. Results characterize the impacts of windows and overhangs on occupants FOVs and potential experiences of window views. Section 5 discusses the potentials of the proposed method based on the results of the pilot study. Section 6 concludes the outcomes of the research and outlines major issues for future developments, especially in terms of daylighting analysis and the quality and visibility of outdoor scenes and elements.

\section{Background: Approaches to View Access Evaluations}

\subsection{Limitations of Existing View Rating Systems}

Existing methods and rating systems for occupants' window views could be improved by considering biological ranges of human vision fields. As summarized in Table 1, 
biological ranges of human eyes' vision fields have not been discussed in existing recommendations and rating systems for view evaluations. More specifically, CEN [1] considers horizontal sight angles to rate individuals' view access to outdoors in terms of minimum (around or above $14^{\circ}$ ), medium (around or above $28^{\circ}$ ), and high (around or above $54^{\circ}$ ). CNE [1] also offers a rule-of-thumb equation and generic graphs to characterize impacts of windows on horizontal sight angles of occupants located in the most remote part of spaces. WELL [21] recommendations for outdoor views are focused on the horizontal distance to transparent envelope glazing and the direct line of sight to outdoors and natural features (Table 1). Vertical view angles of at least $30^{\circ}$ are also recommended to address visibility layers. WELL [21] particularly endorses a view factor of at least 3 based on the view rating system of Heschong Mahone Group [24]. WELL [21] emphasizes on providing such view angles for at least $50 \%$ to $75 \%$ of potential viewpoints throughout the interior space. LEED [22] recommendations for view access are almost similar to WELL [21] in terms of focusing on the direct line of sight and the view factor of 3 and greater based on the rating system of Heschong Mahone Group [24]. More specifically, LEED [22] recommends providing a direct line of sight to outdoors through glazing for at least $50 \%$ of all regularly occupied floor area. LEED [22] also underlines that $50 \%$ of all regularly occupied space should meet at least two of the following options: (i) multiple lines of sight from different directions with about $90^{\circ}$ apart (ii) unobstructed view spotted in three times of the head height of glazing, or (iii) a view factor of 3 or greater based on Heschong Mahone Group [24]. As endorsed by WELL [21] and LEED [22], the view rating system of Heschong Mahone Group [24] provides a scale from 1 to 5 (Table 1), based on the minimum and maximum view angles experienced through horizontal and vertical sights of a seated person towards the window frame of an office. The scale of 5 is assigned to horizontal or vertical view angles of around $50-90^{\circ}$, indicating that the FOVs of the seated person are completely filled by the window frame. The rating scale of 3 refers to $11-15^{\circ}$ view angles, which is still considered as a coherent view. The scales of 2 and 1 are denoted the lowest window view angles which are unacceptable. The scale of 1 is used for view angles of around $1-4^{\circ}$ view angles, indicating a glimpse of a window view to almost blocked FOVs. The Heschong Mahone Group [24] also proposed a gray zone for view angles with in-between scale values which could be rated to a higher or lower scale depending on the content of view. The described methods and rating systems have not considered the characteristics of human eyes' vision fields in experiencing a scene through window frames.

Table 1. Existing rating systems and recommendations for view access to outdoors.

\begin{tabular}{|c|c|c|}
\hline \multicolumn{3}{|c|}{ View rating system offered by CNE in EN-17037 [1] } \\
\hline $\begin{array}{l}\text { Level of recommendation for } \\
\text { view out }\end{array}$ & Horizontal sight angle & \multirow{4}{*}{$\begin{array}{l}\text { Integrating the ranges proposes the following: } \\
\text { High, } 54^{\circ} \geq \text { Medium, } 28^{\circ} \geq \text { Minimum, } 14^{\circ} \geq \text { Unacceptable }\end{array}$} \\
\hline Minimum & $\geq 14^{\circ}$ & \\
\hline Medium & $\geq 28^{\circ}$ & \\
\hline High & $\geq 54^{\circ}$ & \\
\hline \multicolumn{3}{|c|}{ Recommendations for view offered by the WELL [21] } \\
\hline Version 2-Q4 & & \\
\hline
\end{tabular}

- Providing view(s) of natural areas within direct line of sight of at least $75 \%$ of all workstations and seats within conference rooms, lecture halls, or classrooms.

- One of the following requirements is met:

- At least $30 \%$ of the regularly occupied area is within a $20 \mathrm{ft}$ horizontal distance of envelope glazing in each floor and/or in each individual unit.

- At least $70 \%$ of all seating in the spaces is within a $16 \mathrm{ft}$ horizontal distance of envelope glazing.

Version 2-pilot

- Transparent envelope glazing provides access to views for at least 50\% of regular occupants. Views meet at least two of the following requirements:

- $\quad$ View factor of 3 or greater based on Heschong [6]

- Views with a vertical view angle of at least 30 degrees from an occupant facing forward or sideways providing a direct line of sight to the ground or sky. 
Table 1. Cont.

\begin{tabular}{|c|c|c|c|}
\hline \multicolumn{4}{|l|}{ LEED [22] (v4) } \\
\hline \multicolumn{4}{|c|}{$\begin{array}{l}\text { - } \quad \text { Achieve a direct line of sight to outdoors via vision glazing for } 50 \% \text { of all regularly occupied floor area. } \\
\text { - } 50 \% \text { of all regularly occupied floor area must have at least two of the following four kinds of views: } \\
\text { - } \quad \text { Multiple lines of sight to vision glazing in different directions at least } 90 \text { degrees apart; } \\
\text { - } \quad \text { Vnobstructed views located within the distance of three times the head height of the vision glazing; }\end{array}$} \\
\hline \multicolumn{4}{|c|}{ View rating system offered by Heschong Mahone Group [24] } \\
\hline \multirow{2}{*}{ View Rating } & \multicolumn{3}{|c|}{ View Angle (degrees) } \\
\hline & Min-Max & Gray Zone & \\
\hline 1 & $1-4$ & & Unacceptable range (almost blocked) \\
\hline 1 or 2 & & $4-5$ & \\
\hline 2 & $5-9$ & & \\
\hline 2 or 3 & & $9-11$ & \\
\hline 3 & $11-15$ & & $\begin{array}{l}\text { Minimum acceptable threshold } \\
\text { considering the WELL }\end{array}$ \\
\hline 3 or 4 & & $15-20$ & \\
\hline 4 & $20-40$ & & \\
\hline 4 or 5 & & $40-50$ & \\
\hline 5 & $50-90$ & & Highest range \\
\hline
\end{tabular}

The typical biological vision fields offer a fundamental premise to characterize the potential of space in providing almost blocked to good, wide or immersive visual connections to outdoors. The anatomy of human eyes generally offers higher sensitivity and resolutions towards focused FOVs than far-peripheral monocular vision fields [28-31]. As shown in Figure 1, biological studies have characterized the typical vision fields of human eyes in terms of horizontal and vertical FOVs. the horizontal FOV extends to around $180-220^{\circ}$ eccentrically for both eyes [32]. The vertical FOVs extends to about $55-60^{\circ}$ superior and $60-70^{\circ}$ inferior [32]. The focus vision field, hence, corresponds to around $20-30^{\circ}$ in overall vertical and horizontal FOVs, i.e., about $\pm 10-15^{\circ}$ in each direction, where a person could identify texts $[31,33,34]$. The effective vision field is referred to about $40-60^{\circ}$ in overall vertical and horizontal FOVs, i.e., around $\pm 20-30^{\circ}$ in each direction, where people could recognize colors and shapes $[31,33,34]$. The binocular FOV enabling a stereoscopic vision is corresponded to about $120^{\circ}$ horizontal view angles, i.e., about $60^{\circ}$ for each left and right eyes, and $120-130^{\circ}$ vertical view angles, i.e., about $60^{\circ}$ superior and $60-70^{\circ}$ inferior [32]. The monocular horizontal FOV enabling a temporal far-peripheral vision can exceed $\pm 90^{\circ}$ to around $\pm 110^{\circ}$ view angels for each left and right eye as depicted in Figure 1 [28-31]. Wide and immersive views are often enabled through scenes extended from at least $90-100^{\circ}$ to above $120^{\circ}$ and nearly $360^{\circ}$ in the visual fields of an observer [33,34]. Such biological vision fields of observers are widely considered in designing different visual-related settings and adjustments to human ergonomics [31,35], such as conference rooms, theaters, cinemas, virtual realities, screens, and projectors. The vision fields could analogically improve the view rating systems offered by CEN [1] and the Heschong Mahone Group [24]. 

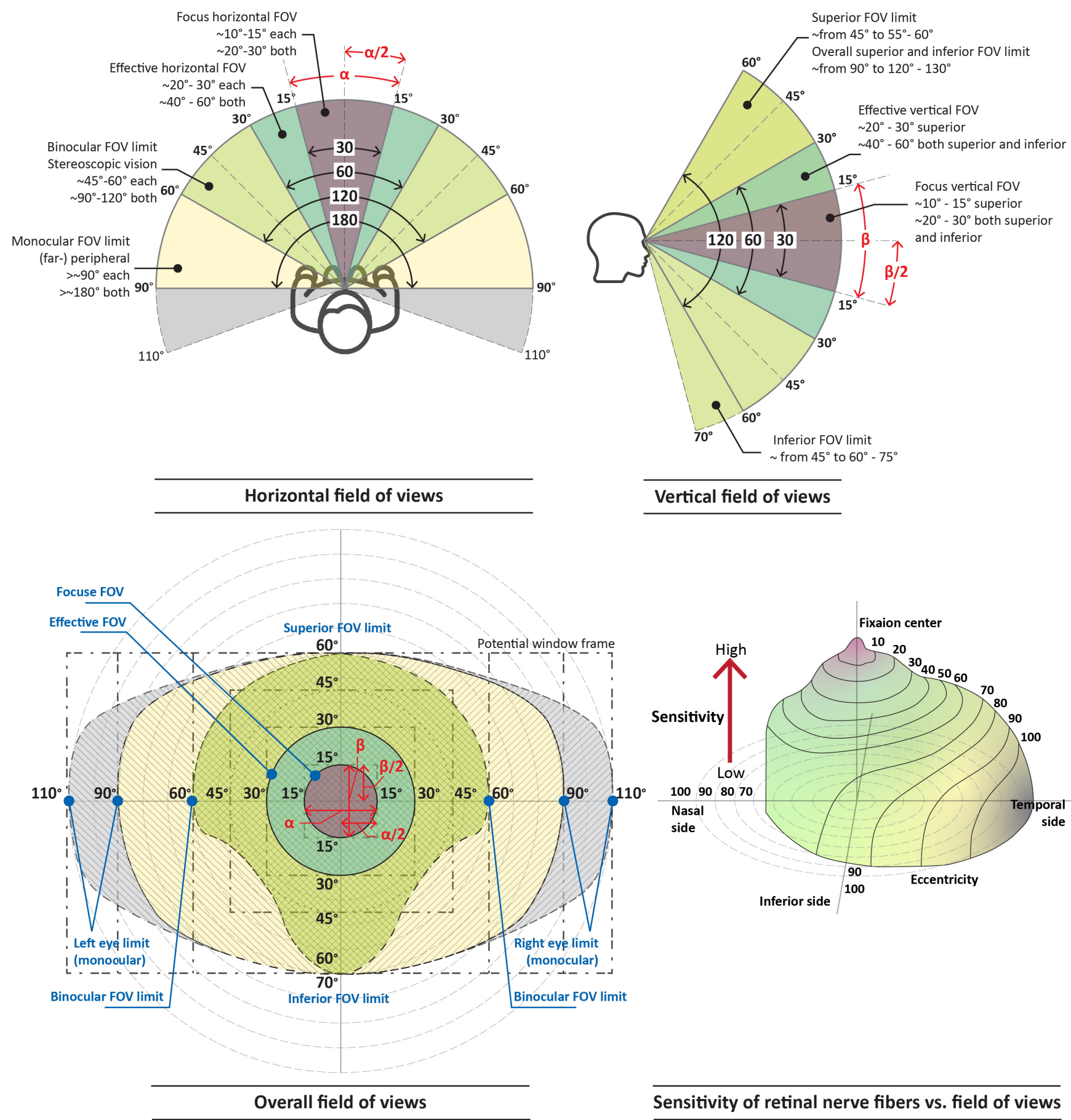

Vertical field of views

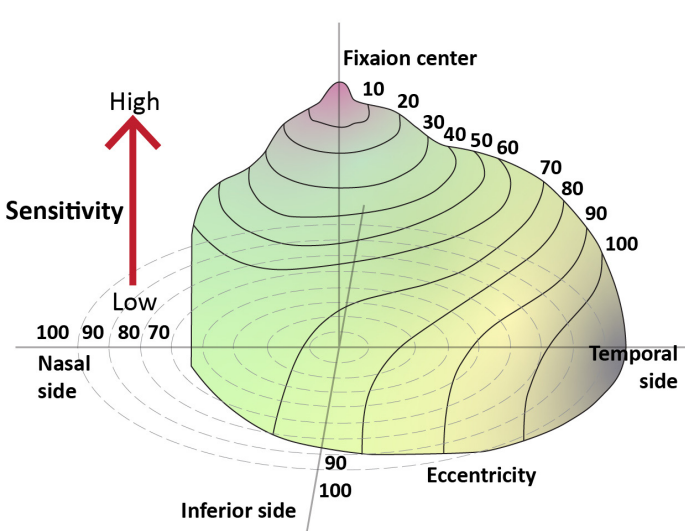

Figure 1. Typical biological vision fields of human eyes and sensitivity of retinal nerve fibers produced based on $[28-30,32,36]$.

The biological vision fields of human eyes could provide a basis to evaluate the overall FOV of occupants towards outdoors. As shown in Figure 1, the overall FOV of an observer is the combination of horizontal and vertical view angles. The overall FOV integrates the impacts of architecture on horizontal and vertical view angels. The horizontal view angle potentially depends on the length or horizontal location of the windows on façades. The vertical view angle potentially depends on the height of observers' eyes, i.e., seated or standing, as well as the height or vertical location of windows. The existing methods and rating systems have not yet discussed the overall FOV of occupants. The approaches are mainly focused on horizontal view angles as the determining factor to evaluate the overall view access of (seated) occupants. CEN [1] and WELL [21] have some general 
recommendations for vertical view angles to discuss visibility layers in terms of viewing outdoor skies or grounds. The view rating system of Heschong Mahone Group [24] also considers the lower view angles, either horizontal or vertical, as the threshold to determine the view rate of an observer. The rating system, thus, potentially masks the visual experiences of higher view angles. For example, the lower scale of 2 is assigned to a viewpoint which has a vertical view angle at scale 2 and a horizontal view angle at scale 4. Evaluating the overall FOV combined with horizontal and vertical view angles could establish a comprehensive framework to characterize architectural impacts on view access potential in space.

\subsection{View Access Computation and Modeling}

Modeling view access in architecture could be improved in terms of efficient computational processes, informative spatial visualizations, and probability analysis. Existing approaches to compute and model view access of occupants have generally employed ray tracing techniques, which are developed for daylighting simulations as in the Radiance engine, or fisheye imagery to evaluate a specific viewpoint in space in terms of recommendations published by CEN [1], LEED [22], or WELL [21]. Evaluating views through ray tracing techniques are used in different modules and research studies, such as Honeybee for Grasshopper of Rhinoceros, Aftab Rad for Autodesk Revit, cove.tool, and Turan, Chegut [20]. In such modules and studies, rays are projected to multiple patches of a 3D sphere considered as a viewpoint. The number of projected rays depends on the preferred resolution, which is accounted for about 7000 in Turan, Chegut [20] and over 20,000 in the Honeybee module assuming a sphere with patches of $20^{\circ}$ by $20^{\circ}$. The ray-tracing-based modules and studies, then, evaluate views based on the percentages of unobstructed rays, presumably in the $120^{\circ}$ of FOVs of a person, that could reach outdoors, particularly reaching an exterior element such as neighborhoods, trees, rivers, or skies. The projected rays are also used to determine the line of sight and the minimum horizontal or vertical view angles to windows, which are required to rate views based on CEN [1], LEED [22], or WELL [21]. Considering imagery techniques, a fisheye image from the viewpoint of an actual or simulated space is generated and post processed in terms of percentages of outdoors or visibility of exterior elements that cover the scene $[25,28]$. Fisheye imagery techniques limit the number of viewpoints while requiring an extensive and time-consuming post processing analysis. Overall, the existing modeling modules mostly focused on specific exterior elements, while insufficient evaluations are given to the accessibility of having a view as well as the characteristic of potential FOVs in space. The computational and modeling process could be optimized by focusing on FOVs towards each window compared to ray tracing approaches and fisheye imagery.

\section{Proposed Method and Parametric Workflow}

\subsection{View Access Integrated with Vision Fields and Biophilia}

This paper offers an integrated computational framework to evaluate window view access by considering the typical biological vision fields of human eyes with the existing rating systems and biophilic recommendations. As a synthase of Table 1 and Figure 1, the horizontal and vertical FOVs of occupants towards outdoors could be evaluated based on the following ranges:

(i) almost blocked to poor views correspond to less than $30^{\circ}$ view angles for overall horizontal FOVs or vertical FOVs, i.e., less than $\pm 15^{\circ}$ view angles in each direction,

(ii) poor to nearly good views correspond to above $30^{\circ}$ and less than $60^{\circ}$ view angles for overall horizontal FOVs or vertical FOVs, i.e., above $\pm 15^{\circ}$ to about $\pm 30^{\circ}$ view angles in each direction,

(iii) good to nearly wide views correspond to above $60^{\circ}$ to around $120^{\circ}$ view angles for overall horizontal FOVs or overall vertical FOVs, i.e., above $\pm 30^{\circ}$ to about $\pm 45^{\circ}$ view angles in each direction. 
(iv) wide to nearly immersive views correspond to above $90^{\circ}$ to around $120^{\circ}$ overall horizontal or overall vertical view angles, i.e., above $\pm 45^{\circ}$ to about $\pm 60^{\circ}$ view angles in each direction which often require eyes' movements to explore.

(v) immersive views correspond to above $\pm 60^{\circ}$ view angles for monocular horizontal FOVs or vertical FOVs, i.e., above $\pm 120^{\circ}$ view angles for the both eyes, which covers the far-peripheral vision field of each eye and eye bulb, or head rotations are required for individuals to see the entire immersed scene.

The horizontal and vertical FOVs could be integrated through the solid angle analysis to enable evaluating the overall FOV of occupants towards outdoors. The overall horizontal and vertical view angles of a person could be analogized as opening angles of a four-sided rectangular pyramid projected from different viewpoints towards the window frame and external overhangs, as the examples in Figure 2. Based on this analogical approach, the solid angle of overall FOVs towards a window could be calculated by using Equation (1). The analogy enables projecting FOVs of a potential observer in various spots towards different architectural configurations of windows and external overhangs (Figure 2). The solid angles of human eyes are also calculated by applying Equation (1) to the analogical pyramid projected in the typical vision fields with a unit distance from a scene. Table 2 displays the calculated solid angles of typical human eyes' FOVs. Figure 3 shows a potential curve fitted to the calculated solid angels of human eyes. The computed solid angles corresponding to each range of human eyes' FOVs could similarly be rated from almost blocked to immersive views. As shown in Table 2, Equation (1) is valid for the maximum horizontal and vertical FOVs towards a single (-side) window, which could be up to $180^{\circ}$ in vertical or horizontal directions, i.e., produces the maximum value of $\sin (\alpha / 2)$ or $\sin (\beta / 2)$. In cases with multiple (-side) windows around or in front of an observer, for example two windows in front or two sides around, the solid angle area could be calculated as the sum of the corresponding values towards each window.

$$
\begin{aligned}
& \Omega(s r)=4 \arcsin [\sin (\alpha / 2) \sin (\beta / 2)] \\
& \alpha, \beta=\text { horizontal and vertical FOVs }
\end{aligned}
$$

Table 2. Calculated solid angles for different vision fields of human eyes.

\begin{tabular}{ccc}
\hline Horizontal View Angle $(\alpha)$ & Vertical View Angle $(\beta)$ & $\Omega(s r)=4 \arcsin [\sin (\alpha / 2) \sin (\beta / 2)]$ \\
\hline $15^{\circ}$ & $15^{\circ}$ & 0.1 \\
\hline $30^{\circ}$ & $30^{\circ}$ & 0.3 \\
\hline $60^{\circ}$ & $60^{\circ}$ & 1.0 \\
\hline $90^{\circ}$ & $90^{\circ}$ & 2.1 \\
\hline $120^{\circ}$ & $120^{\circ}$ & 3.4 \\
\hline $180^{\circ}$ & $130^{\circ}$ & 4.5 \\
\hline $180^{\circ}$ & $180^{\circ}$ & 6.3 \\
\hline
\end{tabular}




\section{FOVs of a potential obsever towards the window frame}

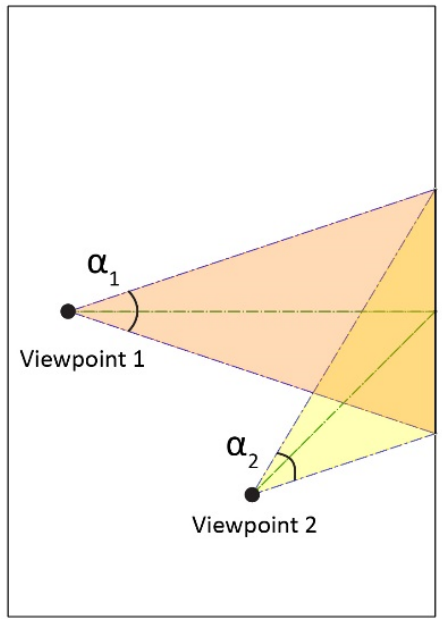

Horizontal Field of View (FOV)

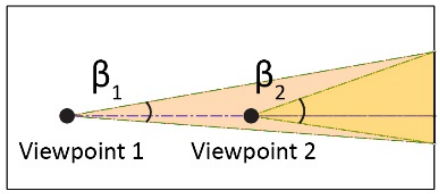

Vertical Field of View (FOV)

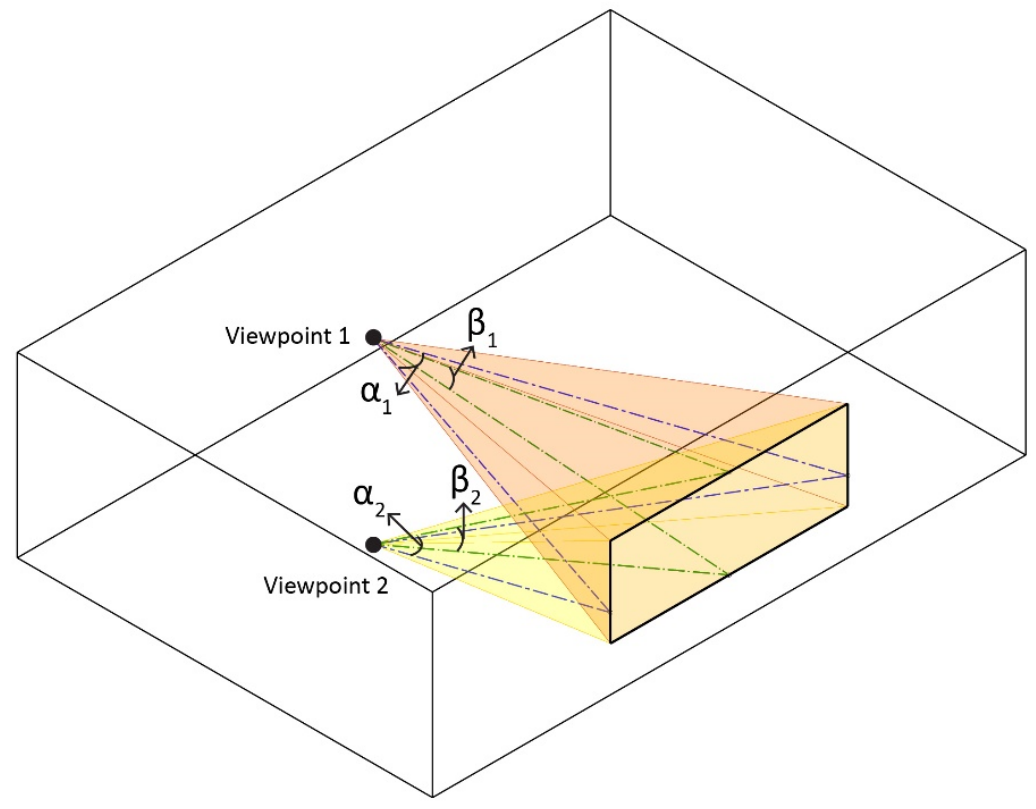

Overall Field of View (FOV)

\section{FOVs' adjustments to the window frame and external overhangs}

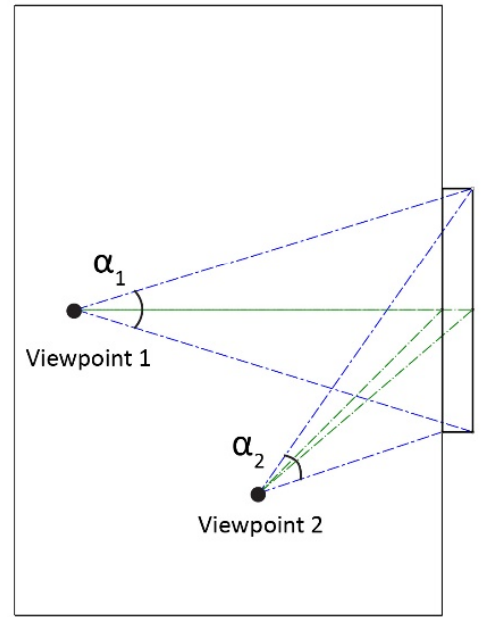

Horizontal Field of View (FOV) adjusted to horizontal overhangs

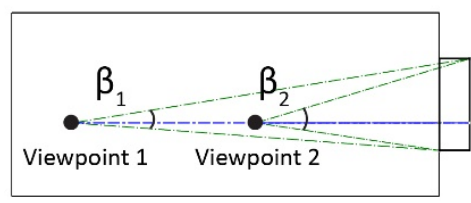

Vertical Field of View (FOV) adjusted to vertical overhangs

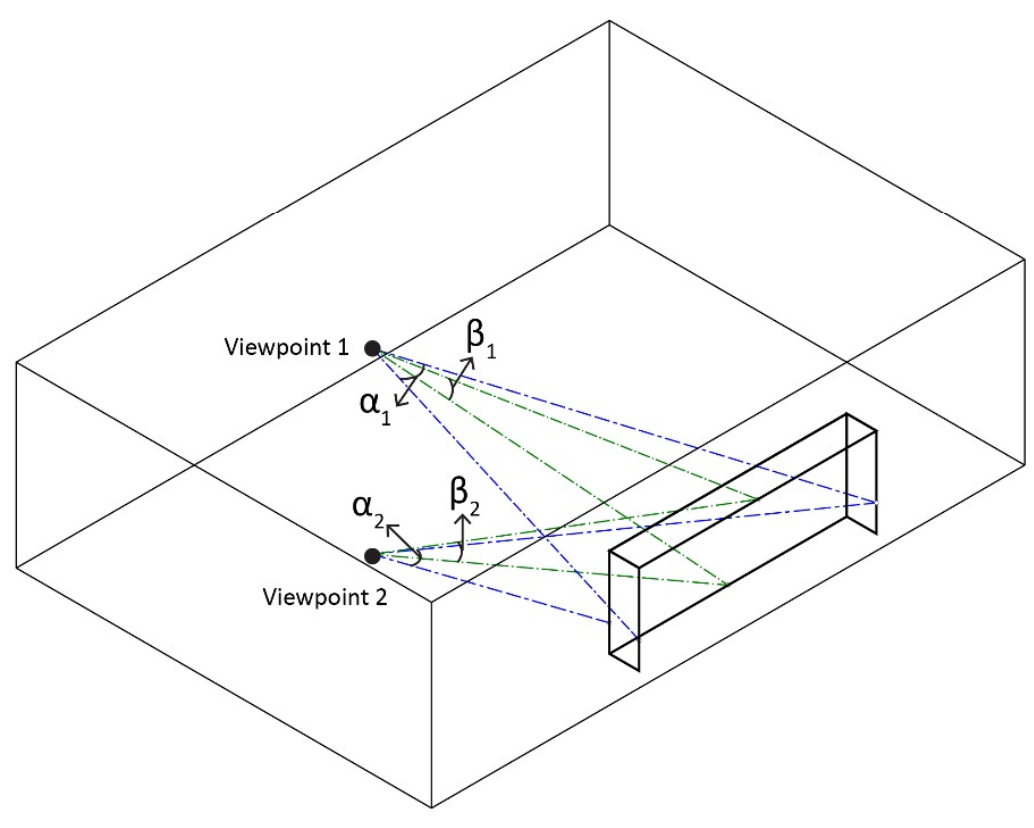

Overall Field of View (FOV) adjusted to overhangs

Figure 2. The overall horizontal and vertical view angles of a potential person with opening angles of a four-sided rectangular pyramid projected from different viewpoints towards the window frame and external overhangs. 


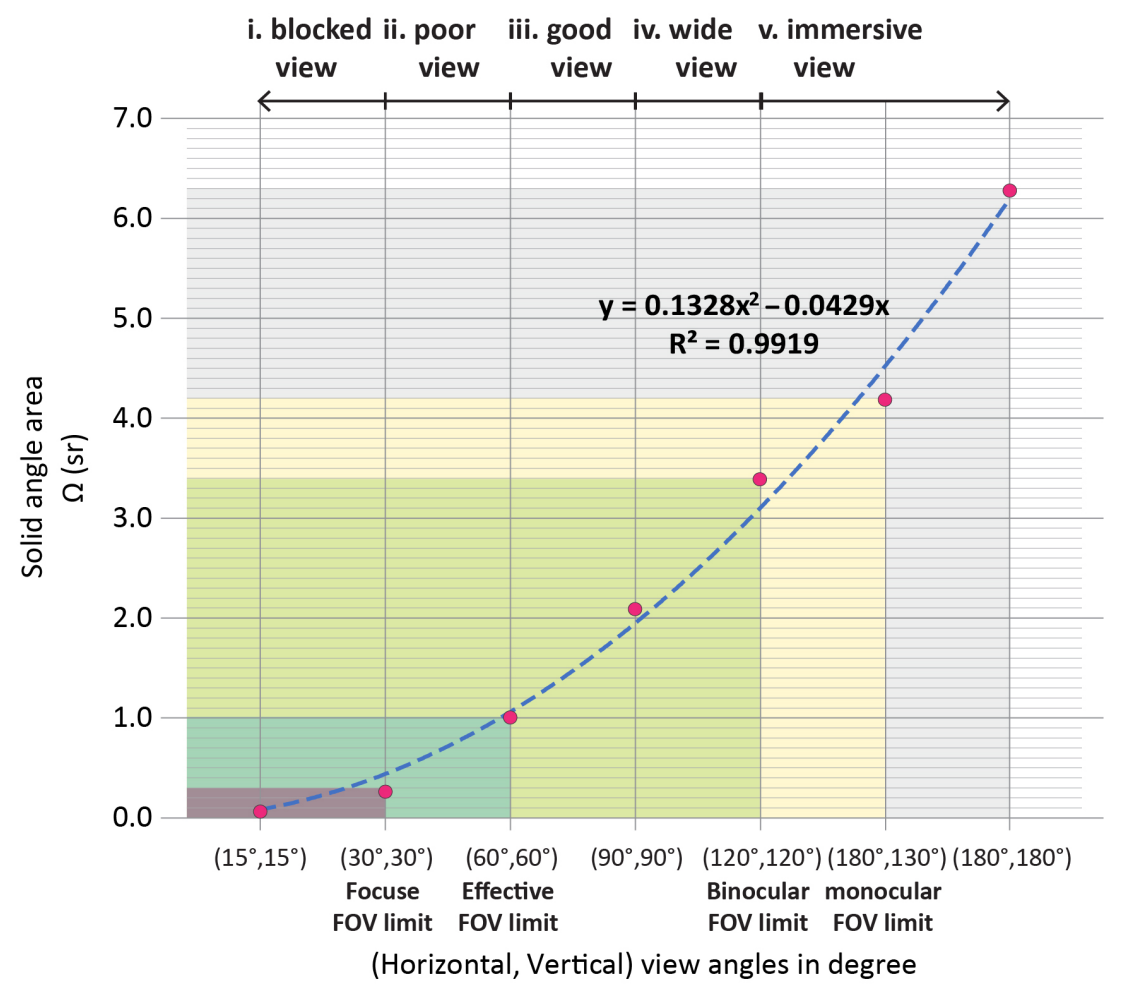

Figure 3. The solid angles with a potential fitted curve calculated for the typical FOVs of human eyes corresponding to almost blocked to immersive views in a unit distance from a scene.

\subsection{Parametric Workflow for Spatial View Access Visualization}

An interactive parametric workflow is developed to automate occupants' view access evaluations and visualization in different architectural configurations based on the proposed computational method and synthesized ranges. As illustrated in Figure 4, the workflow is developed by using the Grasshopper platform of Rhinoceros software coupled with Python scripts. As a pilot study, architectural configurations of a generic space with a single-side window and external overhangs are parametrized in Grasshopper. Figure 5 shows the dimension of the generic space used in the pilot study, i.e., $10 \mathrm{~m}, 7 \mathrm{~m}$, and $3 \mathrm{~m}$ for length, width, and height, respectively. The parametric workflow is briefly described in the following.

\section{Building a parametric architectural configuration}

Values of the parametric variables, such as the length, width, and height of space, windows, or overhangs, are adjustable through sliders or lists assigned.

\section{Generating potential viewpoints}

Horizontal and vertical grid points are generated, which represent potential observers at seated and standing positions throughout the space. Configurations and locations of the horizontal and vertical grid points are interactively adjustable by changing the assigned sliders for the height, width, and length of horizontal or vertical grids as well as the size of the cell on each plane. The centers of grid cells are also automatically determined as the observer's spot in the space. Figure 5 shows the horizontal and vertical grids of viewpoints configured for the pilot study. A total of 280 horizontal viewpoints are rendered as centers of the horizontal grid cells. A total of 70 vertical viewpoints are also generated corresponding to the center of the vertical grid cells.

\section{Computing overall, horizontal, and vertical FOVs}

Horizontal, vertical, and overall view accesses towards outdoors are calculated in terms of horizontal and vertical angles as well as solid angles of FOVs from the generated viewpoints. Considering Figures 2 and 5, horizontal FOVs are computed as the horizontal 
angle from every viewpoint towards the projected intersections of the horizontal grid plane with edges of every window frame or external overhangs. Likewise, the vertical FOVs are computed as the vertical angle created from each viewpoint to the interactions of the projected normal planes and every window frame or external overhang. The calculated horizontal and vertical FOVs are, then, used to compute the overall FOVs of the horizontal viewpoints for a potential seated or standing observer in terms of solid angles by using Equation (1).

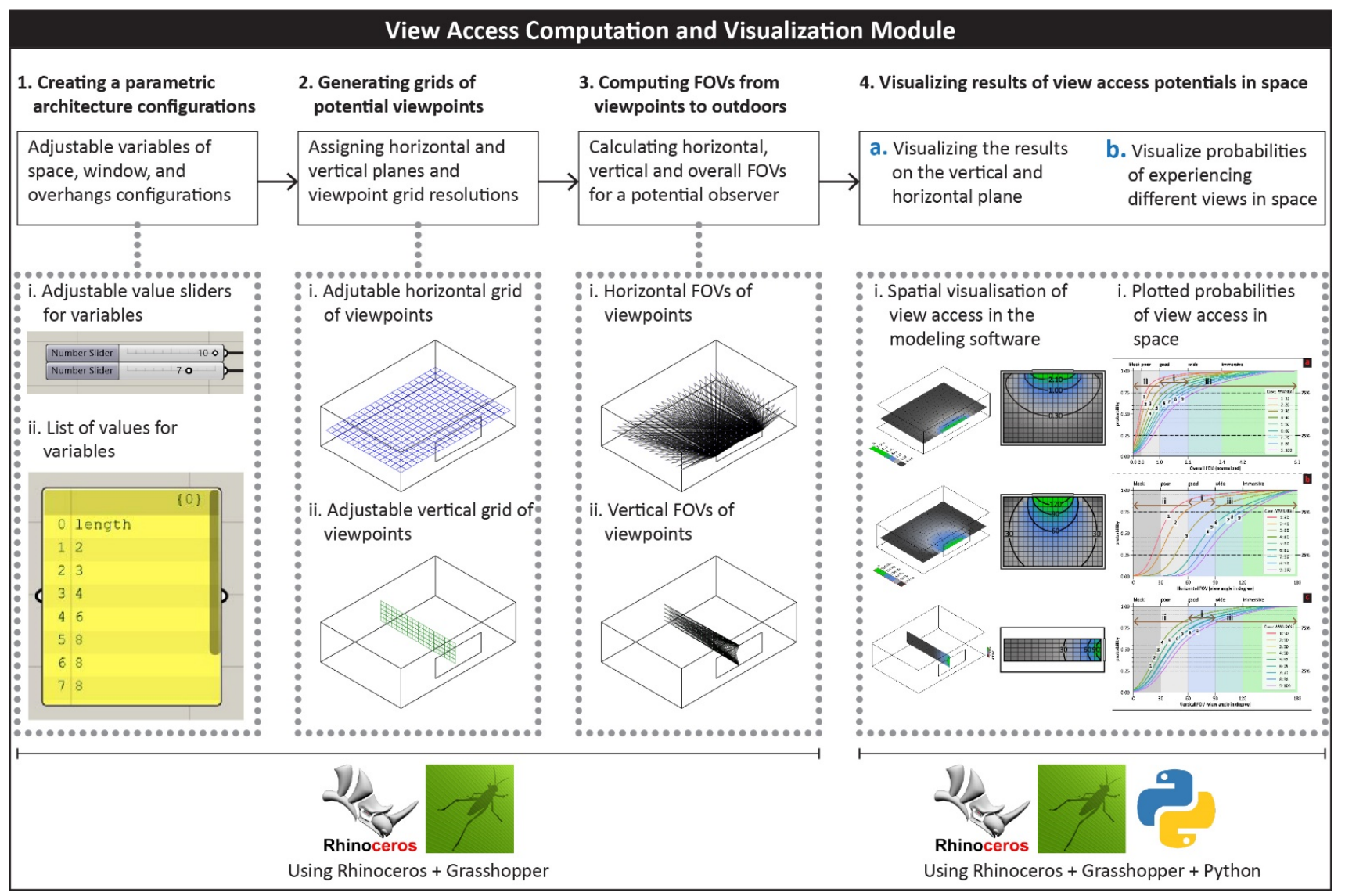

Figure 4. The developed parametric workflow to compute and visualize the view access in space.

\section{Rendering spatial visualization, histograms and cumulative distributions}

Spatial visualizations, histograms, and cumulative probability evaluations are plotted for the computed horizontal, vertical, and overall FOVs. Spatial visualizations of view access are offered in false color maps of overall FOVs (in solid angle steradians) and horizontal and vertical FOVs (in view angle degrees). A python script is developed to render histograms and cumulative distributions of FOVs computed for different design scenarios. The computed FOVs are discrete values which could potentially be non-normally distributed datasets. Histograms present the probability density of different ranges of view access. The cumulative distribution function (CDF) of each computed FOV is also plotted as CDFs could better characterize the general probability pattern of normally or non-normally distributed datasets compared to histograms or probability density functions. The CDF shows the probability of experiencing a specific view range or likelihoods of exposure to less or higher than a specific FOV in the space. The CDF also enables comparing multiple case studies in a single graph. Such potentials of CDFs could help designers and architects to better comprehend the impacts of different design scenarios and parameters on probabilities of experiencing different views in space. The CDF is generally calculated based on Equation (2). This research uses the python library of Seaborn to plot CDFs with fitted curves computed based on a gaussian Kernel Density Estimation (KDE). The libraries 
of Seaborn, Scikit-Learn, SciPy, and Statsmodels are widely recognized as valid tools for data analysis and visualization including CDF plots and gaussian KDE fitted curves.

$$
F O V(x)=\operatorname{Pr}(X \leq x)=\sum_{i \leq x} F O V(i)
$$

Cumulative probability of experiencing a random view of $x$, i.e., the sum of probabilities of all views equal to less than $x$.
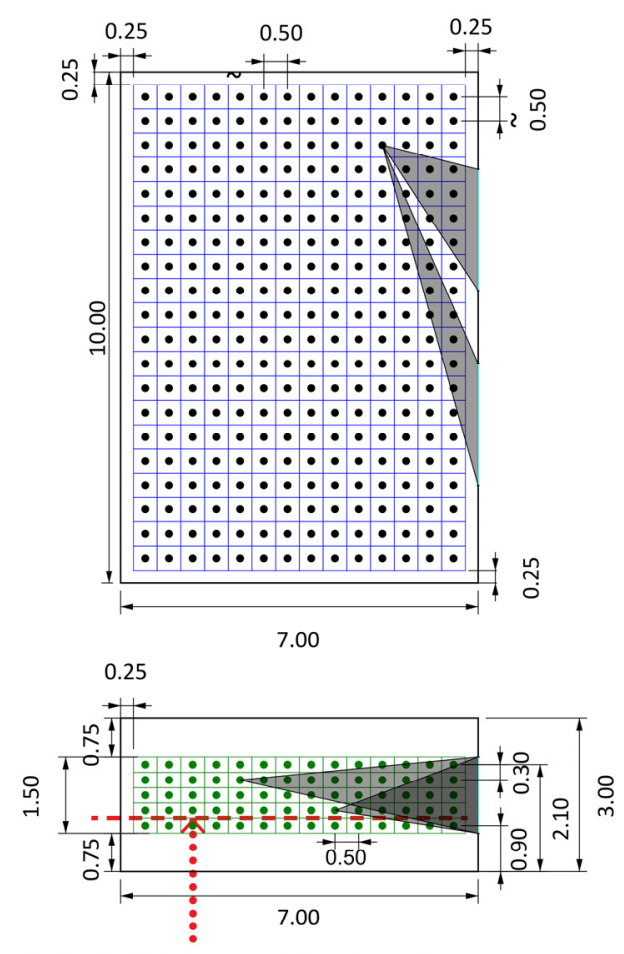

The height of the horizontal plane $(1.20 \mathrm{~m})$ representing potential seated observers for the pilot study.

Figure 5. The configuration of horizontal and vertical grids and viewpoints in the pilot study with examples of view access towards windows for potential seated and standing observers (all dimensions are given in $\mathrm{cm}$ ).

\subsection{View Access Evaluations for Case Studies}

The developed method and parametric workflow are used to evaluate view access in three different architectural design scenarios for windows and external overhangs of the generic space as a pilot study. Figure 6 shows scenarios a, b, and c, proposing 15 different case studies, as described in the following.

- Scenario-a aims at exploring impacts of window size variations on view access of occupants. Occupants' view access is evaluated in nine case studies in terms of window-to-wall ratios (WWR) varied from nearly $10 \%$ to $90 \%$. Results of scenario-a are discussed in Section 4.1.

- Scenario-b aims at evaluating view access impacts of dividing a single large window into multiple smaller windows with a similar overall WWR. A case study with a WWR of $20 \%$ is considered as the reference. The reference case's window is, then, divided from the lengths into two, three, and five multiple smaller windows. The overall WWR and the height of smaller windows are identical to the reference case to enable understanding the impacts of multiple frames. Note that dividing the window size of around and higher than $40 \%$ WWRs produces multiple frames which are almost similar to the single window frame as a small space remains among each frame. Meanwhile, a $10 \%$ WWR window is too small to be divided into multiple smaller window frames. Results of scenario-b are presented in Section 4.2. 
- Scenarios-c aims at characterizing the impacts of external overhangs with different sizes on occupants' view access to outdoors. External overhangs are particularly installed to a reference case with a $60 \%$ WWR in which occupants could potentially experience various view qualities from almost blocked to nearly immersive based on the results of scenario-a. Three different sizes of horizontal and vertical overhangs, i.e., $0.5 \mathrm{~m}, 1.0 \mathrm{~m}$, and $1.5 \mathrm{~m}$, are studied as potential design variations which could affect view access of occupants. Results of scenario-c are explained in Section 4.3.

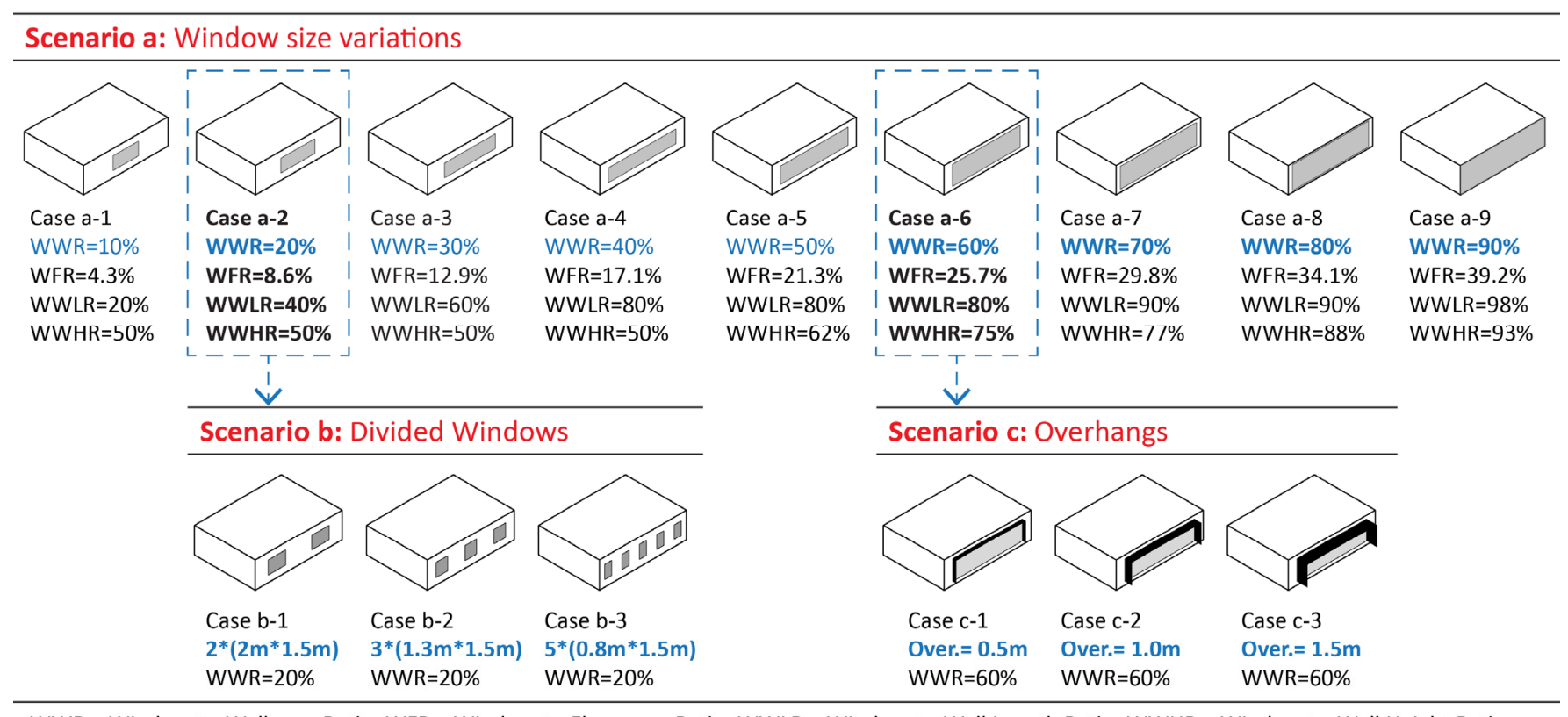

WWR = Window-to-Wall area Ratio; WFR = Window-to-Floor area Ratio; WWLR $=$ Window-to-Wall Length Ratio; WWHR $=$ Window-to-Wall Height Ratio

Figure 6. Architectural design scenarios as case studies for view access evaluations through the developed method and workflow.

\section{Results}

\subsection{Scenario-a: View Access through Different Window Sizes}

Results of computed view access in scenario-a (Figures 7-9) characterize the positive effects of increasing window sizes on occupants' visual connections to outdoors. As shown in the false color maps in Figure 7, window sizes of around 20\% WWR (case a-2) enable a poor view access, accounting for around 0.3 overall FOVs in most of the viewpoints in space. The histograms of the overall FOVs (Figure 8) illustrate the high probability densities of almost blocked and poor views i.e., about 75\%, in cases a- 1 to a-3, having WWRs of $30 \%$ or less. Occupants of such spaces experience less than $60^{\circ}$ horizontal FOVs and $30^{\circ}$ vertical FOVs from most viewpoints in space, especially in locations far from the window. The histograms of horizontal and vertical FOVs, provided in Appendix A-Figures A1 and A2, reveal the higher densities of experiencing such low-angle views in cases a-1 to a-3. A few spots close to the window enable good view access to outdoors, with above 1.00 overall FOVs, $60^{\circ}$ horizontal FOVs, and $30^{\circ}$ vertical FOVs. The probability densities of such views are less than $5 \%$ (Figure 8 ). Increasing the window length has positive effects on view access. As rendered in Figure 7, cases a- 4 to a-9, increasing WWRs from $40 \%$ to $90 \%$ provides larger horizontal FOVs of above $60^{\circ}$ over the space, and above $120^{\circ}$ around the window. False color maps visualize the contributions of higher window heights to enable larger vertical FOVs of above $30^{\circ}$ at the individuals' eye level from most viewpoints in space. As further shown by histograms in Figure 8, increasing WWRs reduces the probability densities of almost blocked and poor overall FOVs to around $50 \%$ or less, while it improves the probabilities of experiencing good, wide, and immersive views in space. 


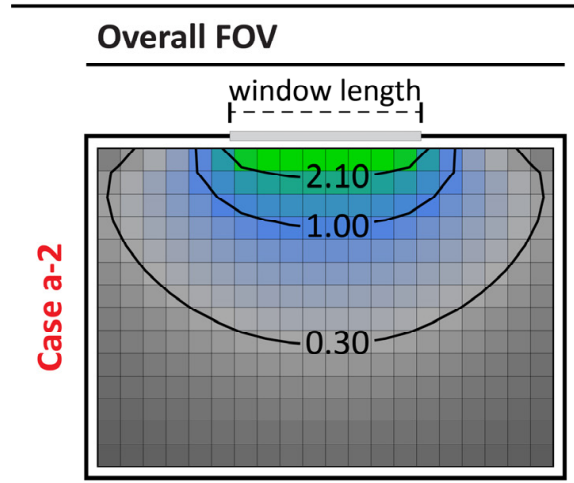

window length

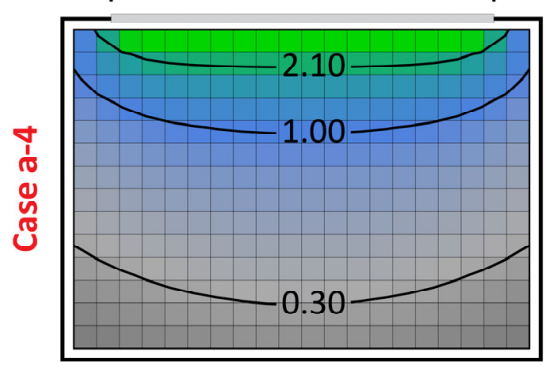

window length

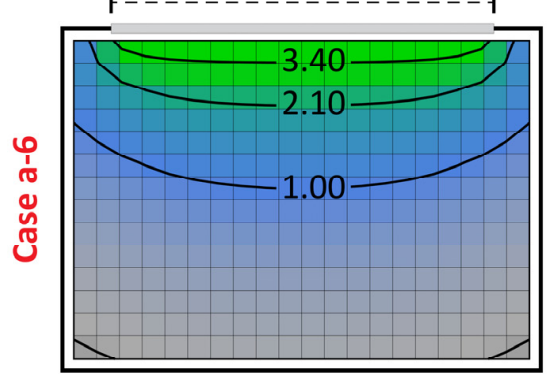

window length
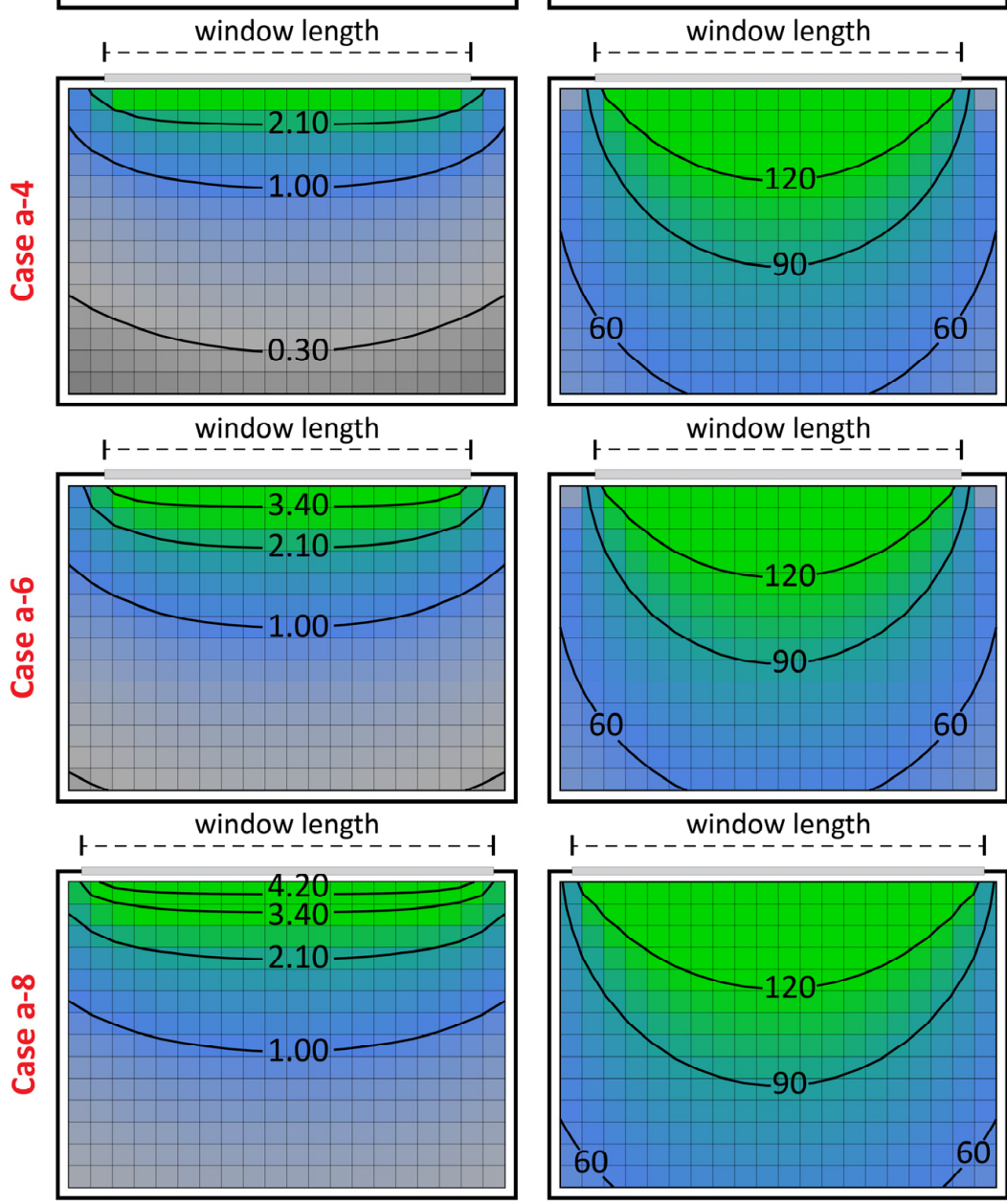

window length

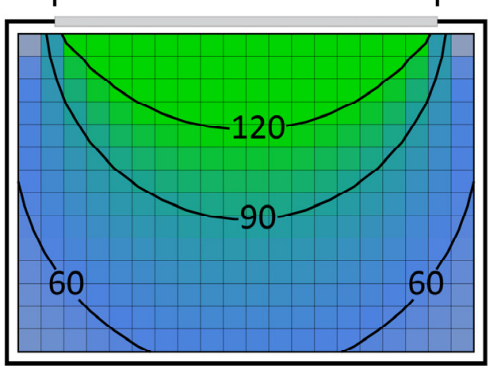

window length

\section{Vertical FOV}

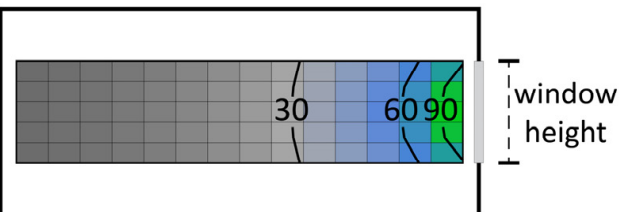

WWR $=20 \%$

WFR $=8.6 \%$

WWLR $=40 \%$

WWHR $=50 \%$

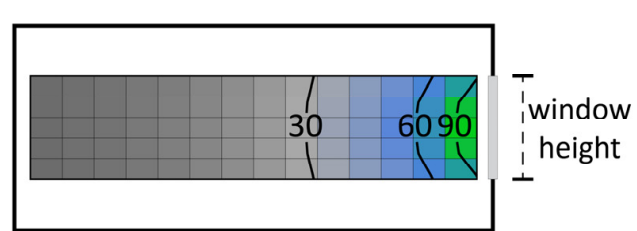

WWR $=40 \%$

WFR $=17.1 \%$

WWLR $=40 \%$

WWHR $=50 \%$
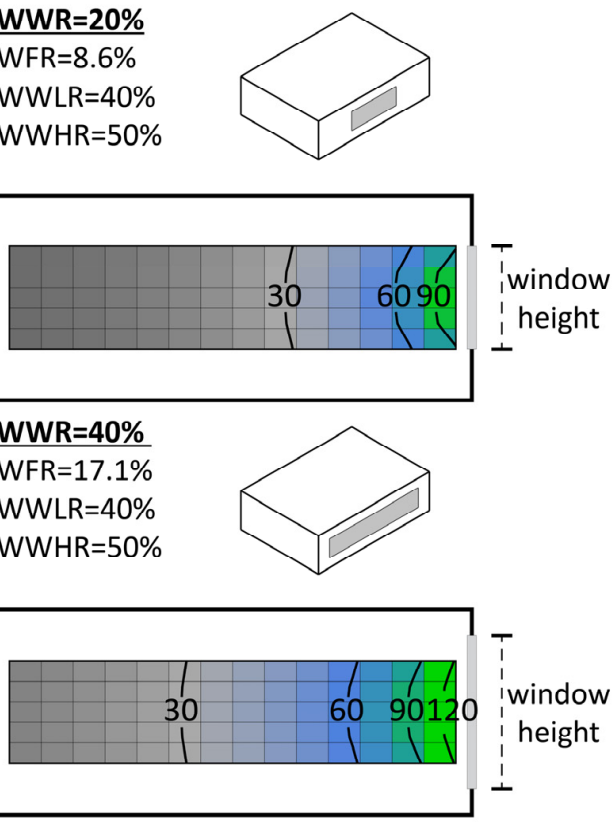

WWR $=60 \%$

WFR $=25.7 \%$

WWLR $=80 \%$

WWHR $=75 \%$
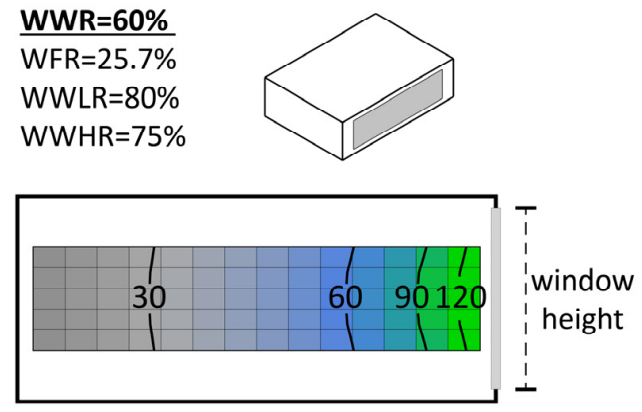

WWR $=80 \%$

WFR $=34.1 \%$

WWLR $=90 \%$

WWHR $=88 \%$

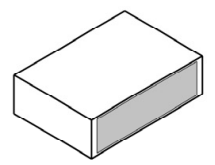

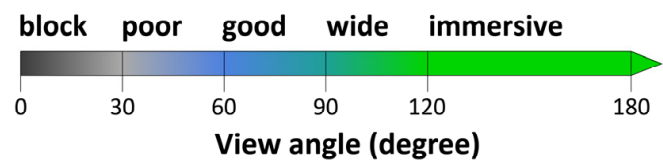

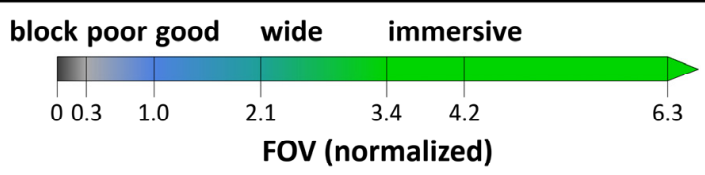

Figure 7. Examples of view access in false color maps in terms of computed overall, horizontal, and vertical FOVs in scenario-a, including case studies with different window sizes. 


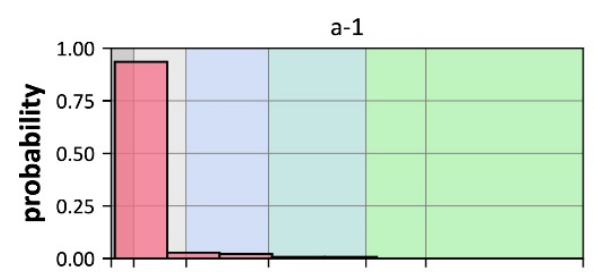

a-4

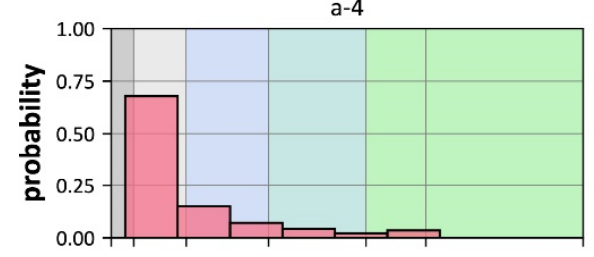

a-7

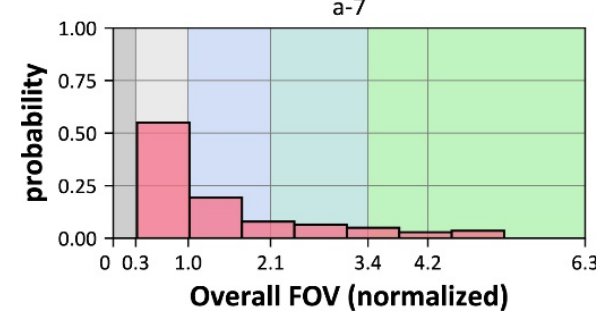

a-2

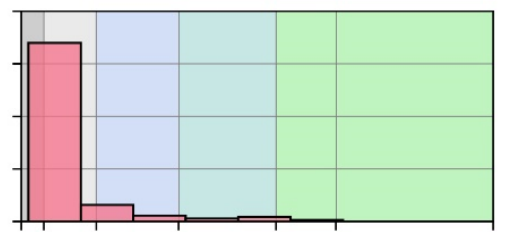

a-5

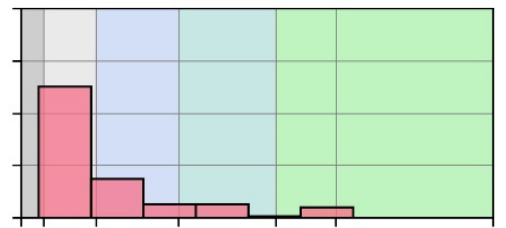

a-8

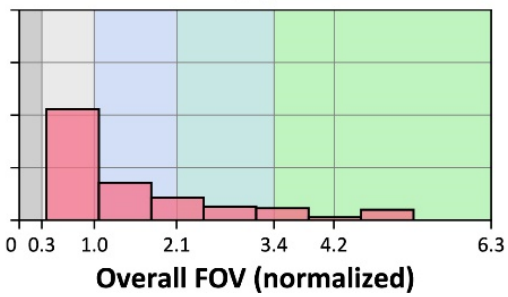

a-3

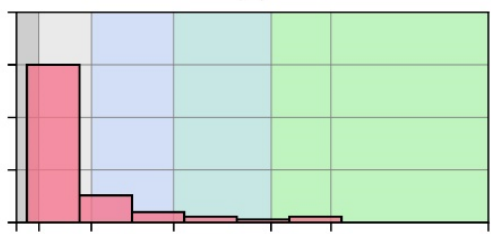

a-6

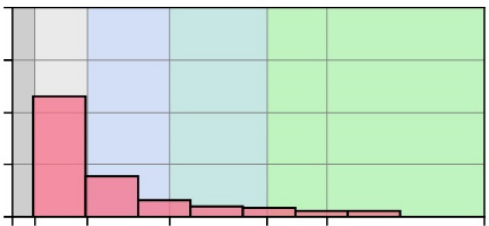

a-9

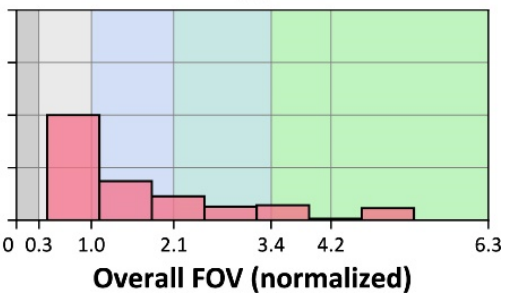

Figure 8. The histograms of the computed overall FOVs for scenario-a, including case studies (a-1) to (a-9) with different window sizes.

The cumulative probabilities of computed FOVs for scenario-a (Figure 9) further reveal positive contributions of increasing window sizes to experience a biophilic view access to outdoors. Considering the overall FOV patterns in Figure 9-1, occupants have 95\% to $75 \%$ probabilities to experiencing poor to nearly blocked views, i.e., less than 1.0, in cases a-1 to a-3 offering $15 \%$ to $30 \%$ WWRs. Likelihoods of almost blocked views, i.e., less than 0.3 , are about $60 \%$ to $30 \%$ in such cases. Cases a- 1 to a-3 also offer occupants less than $5 \%$ to $20 \%$ likelihoods to experience good to wide view access, i.e., above 1 . Likelihoods of experiencing poor to almost blocked views reduce to $70 \%$ to $40 \%$ by increasing WWRs to $40 \%$ and $90 \%$ enabled in cases a- 4 to a-9. Such window sizes also decrease probabilities of almost blocked view access, i.e., less than 0.3 , to about $25 \%$ to $10 \%$ throughout space. Occupants of cases a- 4 to a- 9 have about $40 \%$ to $60 \%$ probabilities to be exposed to good, wide or immersive views, i.e., above 1.0. The view access probability pattern of case a- 8 is relatively similar to case a-9. Individuals also have about $10 \%$ to nearly $30 \%$ likelihoods to experience wide to immersive views, i.e., above 2.1 , by enabling $40 \%$ to $90 \%$ WWRs in cases a- 4 to a-9. Focusing on cases a-7 and a-9, the CDF patterns are fairly similar, i.e., with less than $5 \%$ to $10 \%$ differences. WWRs increase from $70 \%$ to $90 \%$ in cases a- 7 to a-9 while probabilities of having poor to almost blocked views improve less than $10 \%$ from nearly $50 \%$ to $40 \%$. The likelihood of experiencing almost blocked views in cases a-7 to a-9 are around $15 \%$ to $10 \%$. The cases also offer close probabilities of experiencing good to wide views, i.e., also around $50 \%$ to $60 \%$, and wide to immersive FOVs, i.e., around $20 \%$ to $25 \%$. Therefore, WWRs of around $40 \%$ to $70 \%$ offer an optimum range to experience biophilic view access to outdoors. Occupants have higher potentials to experience good and wide views than poor and blocked FOVs (segment iii vs. segments $i$ and ii in Figure 9-1) in cases a- 4 to a-7, i.e., WWRs of $40 \%$ to $70 \%$ compared to cases a- 1 to a-3, i.e., less than $40 \%$ WWRs. Reducing window sizes to less than $40 \%$ could significantly block viewpoints in the space (segments i and ii). Increasing window sizes to higher than $70 \%$ have less than $10 \%$ contributions to experience more good, wide or immersive views in space (segments iii, iv and $v)$. 

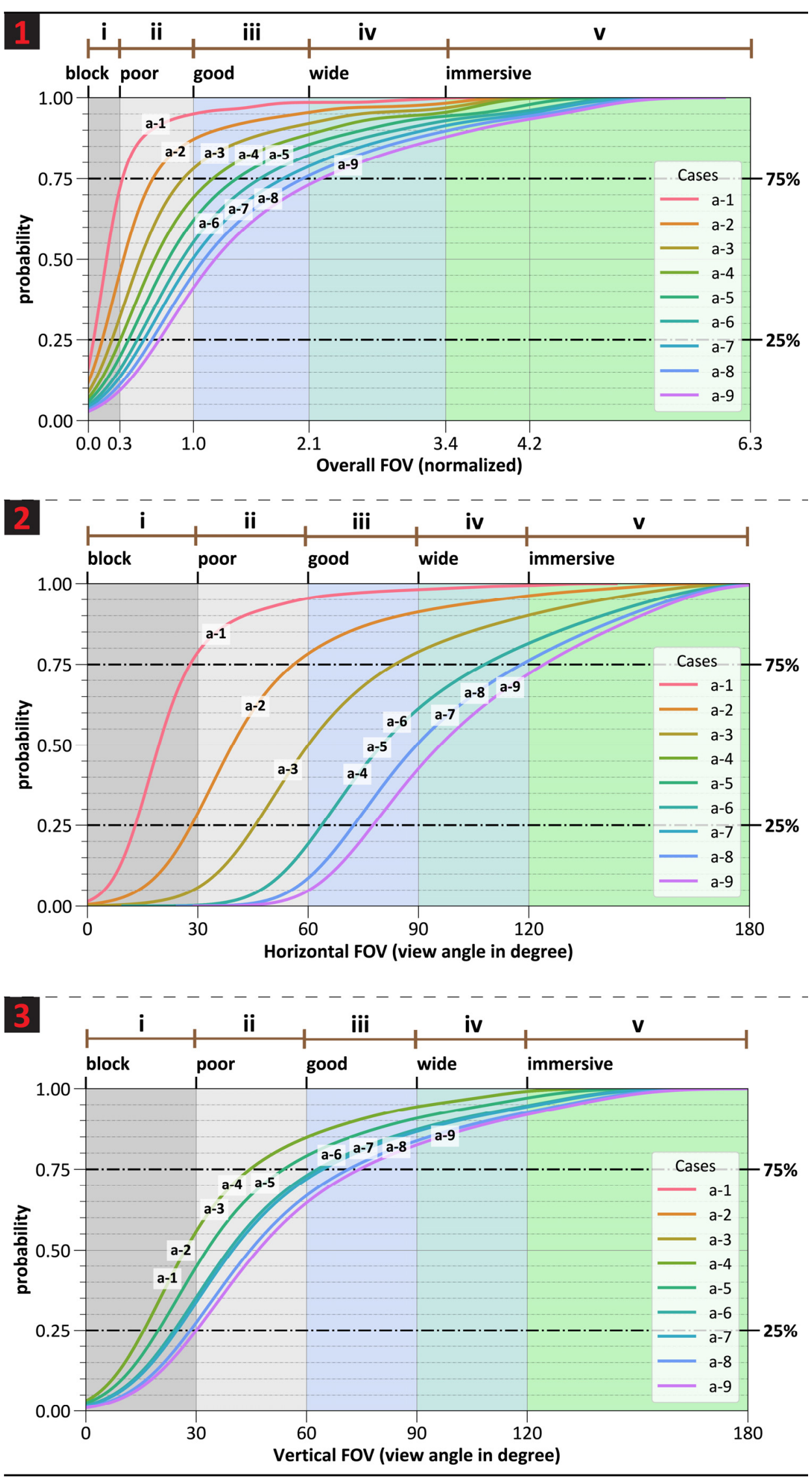

Figure 9. The CDFs of computed overall (9-1), horizontal (9-2), and vertical (9-3) FOVs for scenario-a, including case studies of a-1 to a-9 with different window sizes. 
Considering the horizontal FOV patterns in Figure 9-2, small window lengths of 40\% WWLRs or less in cases a- 1 and a-2 enable a $75 \%$ likelihood of poor to almost blocked FOVs, i.e., less than $60^{\circ}$ view angles. Cases a- 1 and a-2 also enable about $50 \%$ to $30 \%$ likelihoods of almost blocked FOVs, i.e., less than $30^{\circ}$ view angles. Experiencing good to wide horizontal views, i.e., above $90^{\circ}$, is less than $20 \%$ to $10 \%$ in such cases. Increasing the window length to between $40 \%$ to $80 \%$ WWLRs, e.g., around $60 \%$ in case a-3, enable a $75 \%$ likelihood of experiencing good to poor and almost blocked horizontal views, while the likelihood of almost blocked viewpoints is less than 5\%. The 60\%-WWLR case also enables a likelihood of around $20 \%$ of experiencing good to wide and immersive horizontal views. Increasing the window length to $80-100 \%$ WWLRs, such as cases $4-9$, provide higher horizontal view angles over the space, with $75 \%$ likelihoods of immersive (above $120^{\circ}$ ) and wide to poor FOVs. Less than $20 \%$ to $5 \%$ of viewpoints offer poor horizontal FOVs in such cases, while likelihoods of almost blocked horizontal FOVs are zero, i.e., no viewpoint has almost blocked view angles.

The CDFs of vertical FOVs (Figure 9-3) show that cases a- 1 to a-5 with window lengths of around $50 \%$ to $60 \%$ WWHRs offer $75 \%$ likelihoods of poor to almost blocked vertical FOVs, i.e., less than $60^{\circ}$ view angles. The likelihoods of almost blocked vertical views, i.e., less than $60^{\circ}$ view angles, are between $40 \%$ to $60 \%$ with such window heights. Occupants have less than $20 \%$ to $10 \%$ probabilities to experience good or wide views in cases a- 1 to a- 5 . Increasing the window height to above $70 \%$ to $90 \%$ WWHRs, i.e., cases a- 6 to a-9, provide almost similar FOV probability patterns, with $75 \%$ likelihoods of experiencing wide and good to poor and almost blocked vertical views. As shown in Figure 9-3, the likelihoods of almost blocked vertical view access are less than $35 \%$ to around $20 \%$ for window heights of $75 \%$ WWHRs and above. Such cases also enable around $40 \%$ to $25 \%$ likelihoods of good to wide and immersive vertical views throughout space.

\subsection{Scenario-b: View Access through Divided Windows}

Results of scenario-b visualize the impacts of dividing a single window to multiple smaller windows on the distribution of overall and horizontal view access in space. The computed false color maps in Figure 10 reveal that multiple windows can uniform the distributions of overall and horizontal FOVs compared to the corresponding maps for a single window. The renders of the reference case show that good and wide or immersive viewpoints are distributed near the single window. Poor and almost blocked viewpoints are distributed around the corners and back and side walls away from the window. Designing multiple windows, i.e., cases b-1 to b-3, change the FOVs' distribution in space where viewpoints at the corners and back and side walls offer good to wide overall and horizontal views. Viewpoints around the windows also have good view access to outdoors. Yet, the computed view access reveals that divining a single window to very small configurations, such as case b-3 with five window frames, could reduce horizontal FOVs of the viewpoints near the windows from wide and immersive to good. Such changes in view access patterns are also shown in the probability densities of horizontal FOVs' illustrated in Figure 11 and Appendix A-Figure A3. The horizontal FOVs' histograms of cases b-1 to b-3 (Figure 11) show probability densities of almost blocked and low view angles are reduced, while the probabilities of poor to good views are increased compared to the case Ref. There is zero probability density for wide or immersive view in case b-3. The probability densities of overall FOVs (Appendix A-Figure A3) are similar for cases Ref, b-1 to b-3. 
Scenario $b(W W R=20 \% ; W F R=8.6 \%, W W L R=40 \%)$

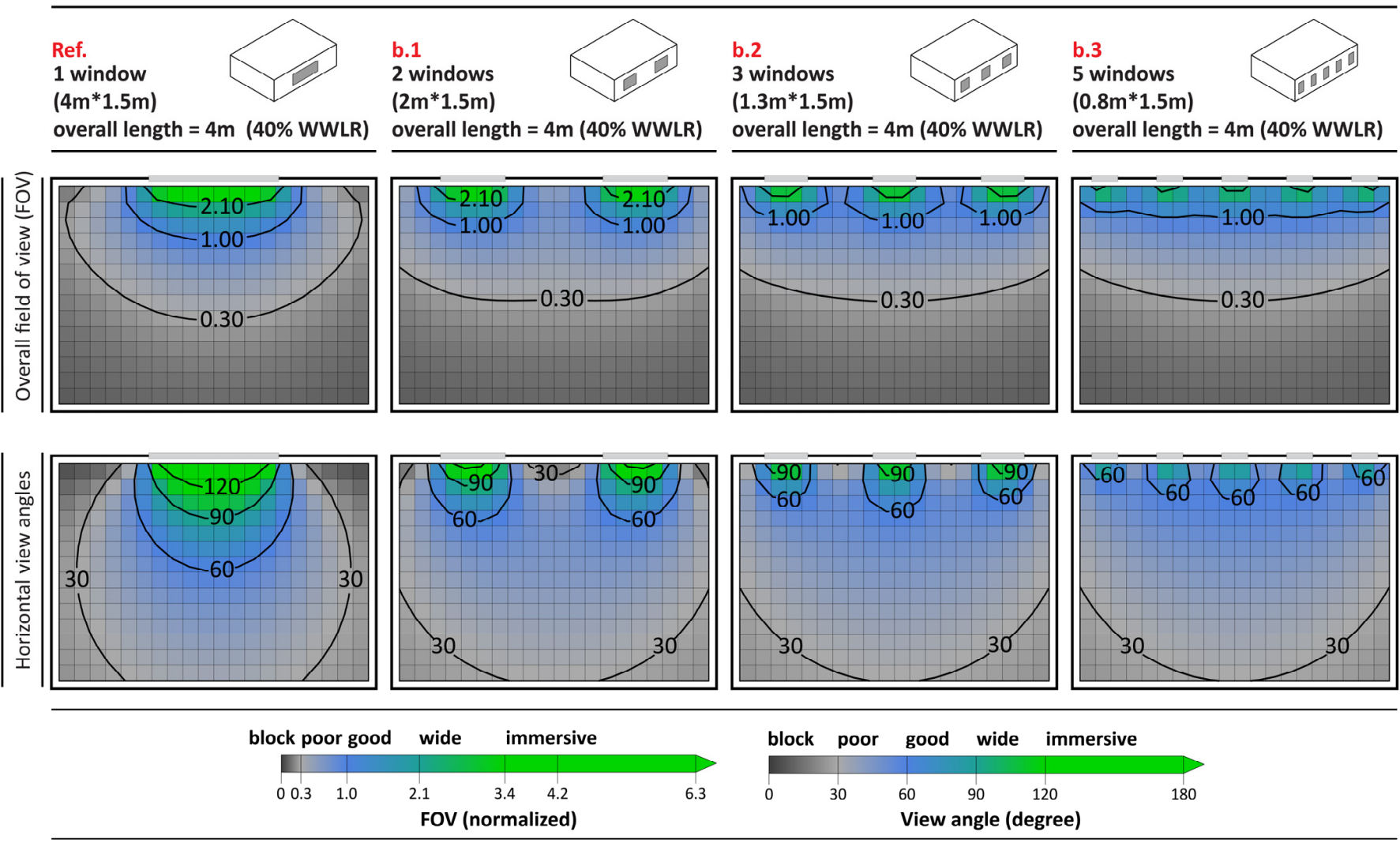

Figure 10. Impacts of dividing to multiple windows on the distributions of overall and horizontal FOVs for the case studies of scenario-b.
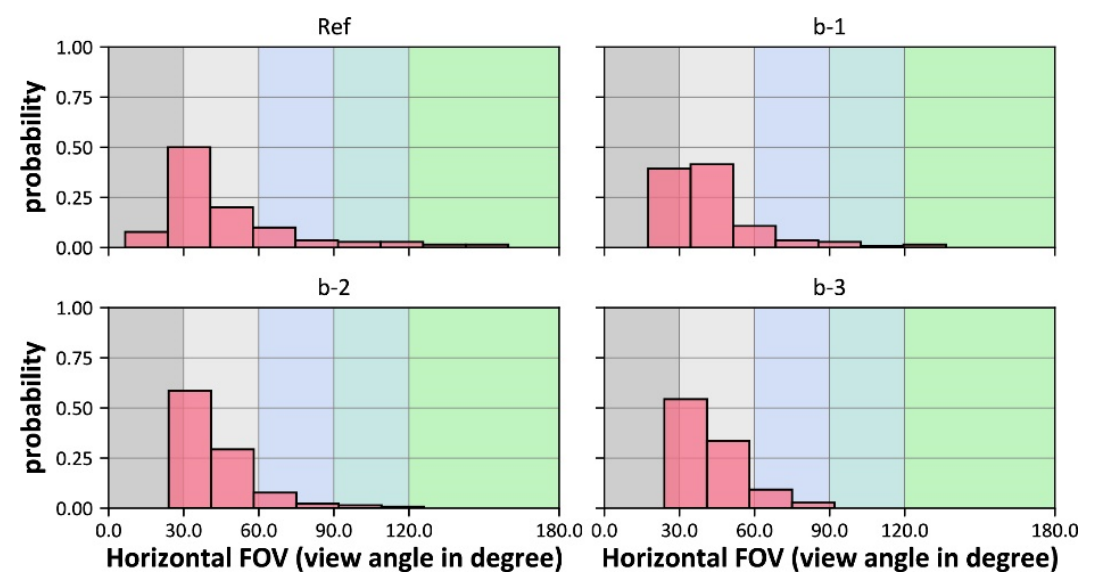

Figure 11. Impacts of dividing to multiple windows on histograms of horizontal FOVs for scenario-b including case studies of Ref. b-1 to b-3.

The cumulative probabilities of FOVs further highlight impacts of divided windows on view access patterns in the space. As illustrated in Figure 12-1, the probability patterns of the overall FOVs for multiple windows are similar to the corresponding single window. Considering Figure 12-2, splitting a single window to multiple windows could improve horizontal FOVs' probabilities by decreasing the likelihoods of almost blocked views by almost $10 \%$ (segment i). However, multiple window scenarios reduce the probabilities of experiencing good and wide views (segments iii and iv) for about $15 \%$ in cases b- 1 to b-3 compared to the corresponding reference case with a single window. 


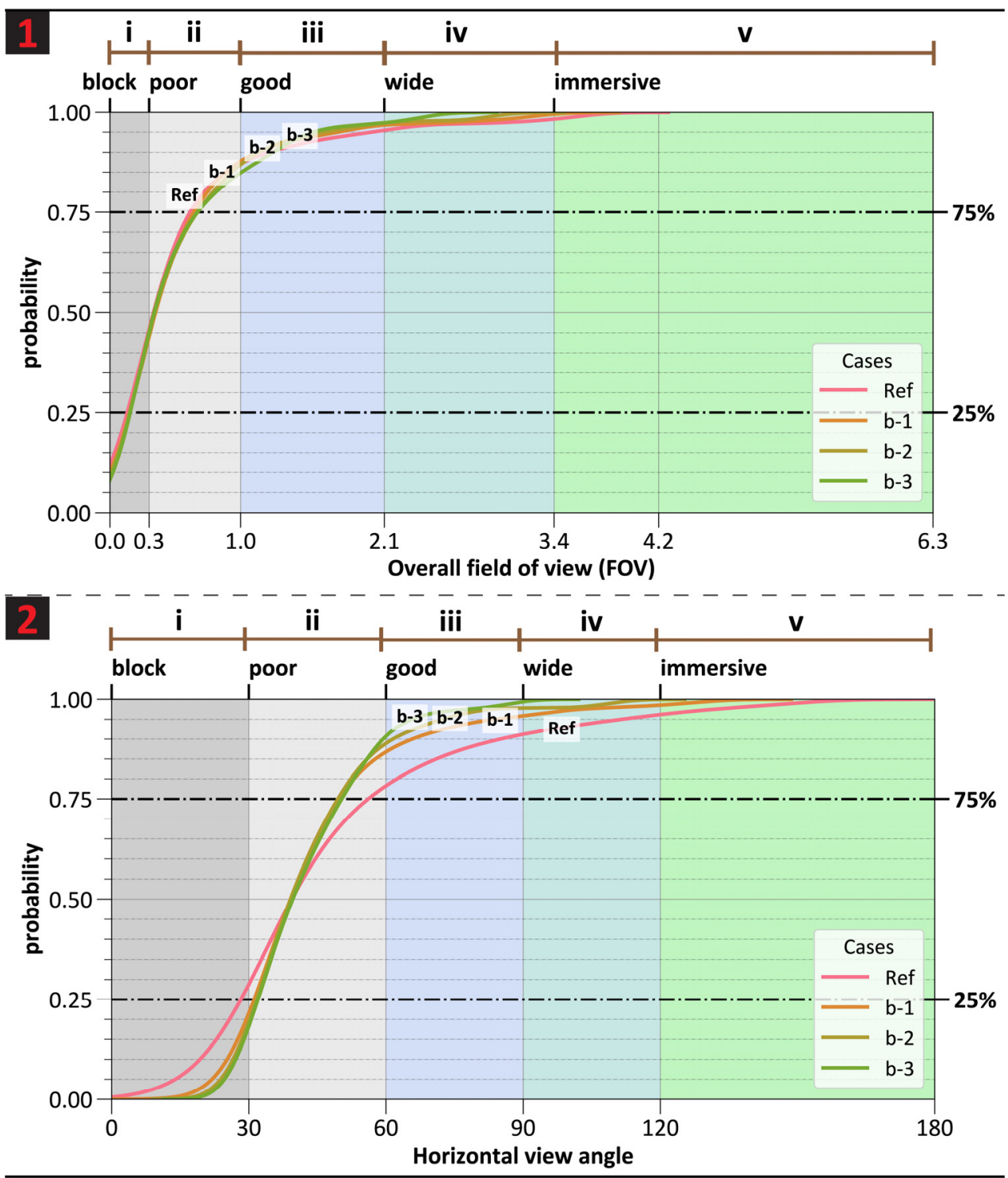

Figure 12. Impacts of dividing to multiple windows on the cumulative probabilities of overall and horizontal FOVs for the case studies of scenario-b.

\subsection{Scenario-c: View Access through Overhangs}

Results of scenario-c show that external overhangs installed on the window edges, i.e., cases c-1 to c-3, reduce the horizontal, vertical, and overall FOVs of viewpoints compared to the reference case. Considering the false color maps of overall FOVs in Figure 13, overhangs reduce the overall view access throughout the space, especially for viewpoints far from the window close to the back wall. Exterior overhangs reduce the horizontal and vertical FOVs of seated or standing individuals in different spots from near the window to the back and side walls comparing the reference case with cases c- 1 to $c-3$. The false color maps of the horizontal and vertical FOVs also show that overhangs have higher negative impacts on the view angles of standing individuals with eye levels at around $1.50 \mathrm{~m}$ to $2.00 \mathrm{~m}$ above the floor compared to seated occupants with eye levels at about $1.00 \mathrm{~m}$ to $1.20 \mathrm{~m}$ above the floor. Increasing the size of external horizontal overhangs from $0.5 \mathrm{~m}$ in case c- 1 to $1.50 \mathrm{~m}$ in case c-3 exacerbates the negative impacts of reducing the overall, horizontal, and vertical FOVs. Negative impacts of external overhangs on view access are also recognizable in the histograms of the overall, horizontal, and vertical FOVs presented in Figure 14 and Appendix A-Figures A4 and A5. The histograms reveal that installing external overhangs 
reduces the probability densities of experiencing wide and immersive views towards good and poor overall, horizontal, or vertical FOVs in space.

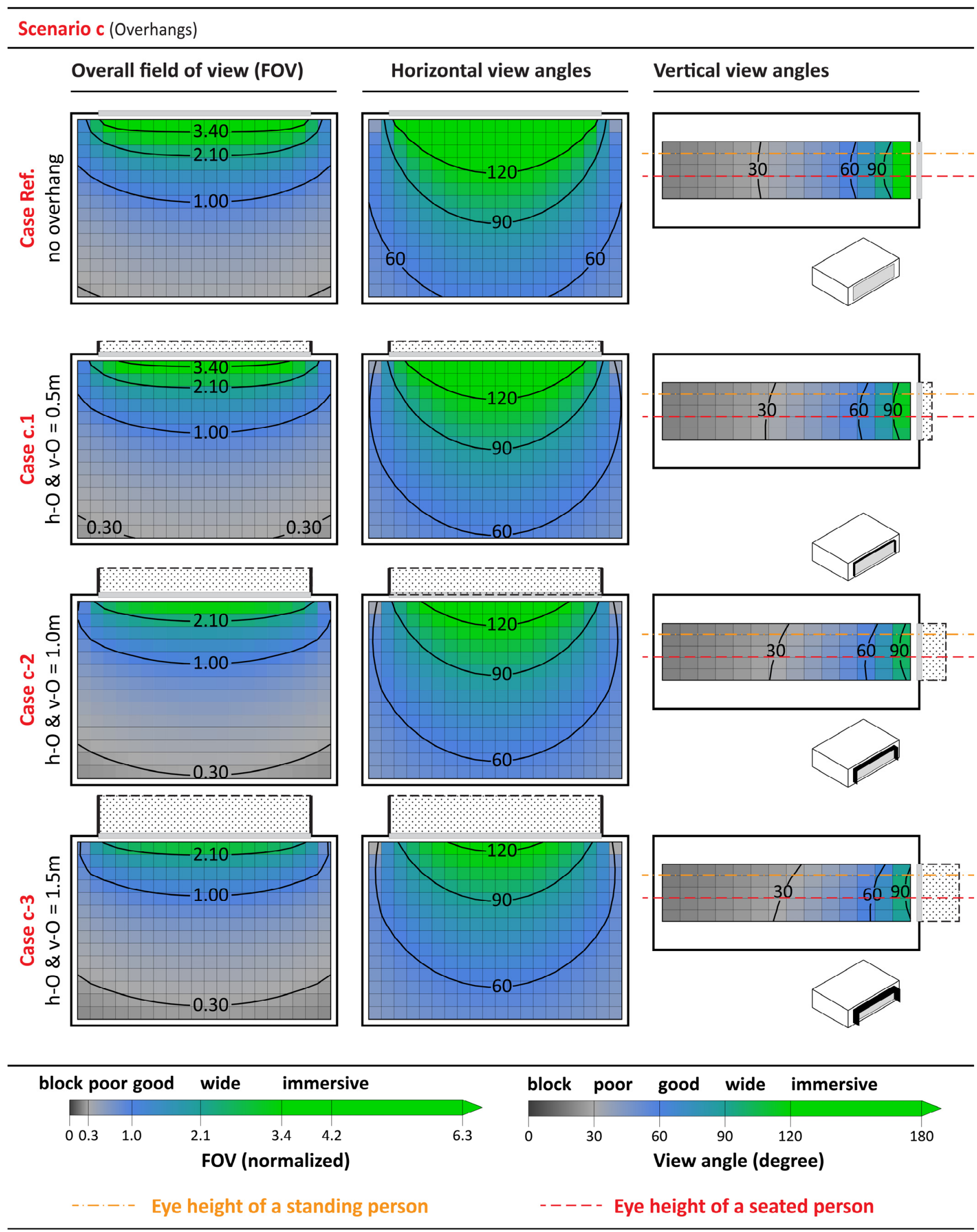

Figure 13. Impacts of external overhangs installed around window edges on the distributions of overall, horizontal, and vertical FOVs for case studies of scenario-c. 


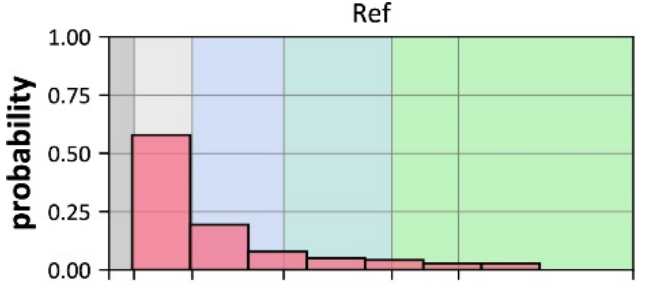

C-2

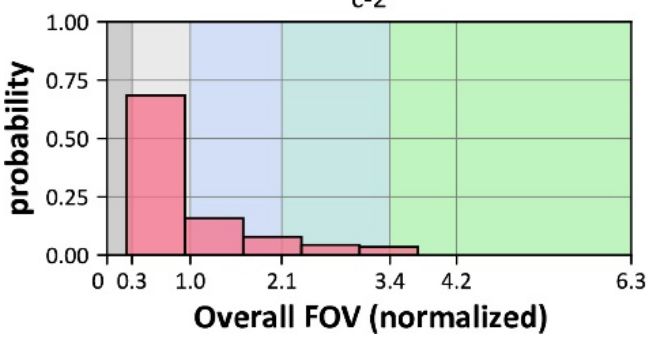

Figure 14. Impacts of external overhangs installed around window edges on the histograms of overall FOVs for scenario-c. including case studies of Ref and c-1 to c-3.

View access impacts of overhangs are better characterized by cumulative probabilities. As illustrated in Figure 15-1, likelihoods of experiencing poor to almost blocked overall views increase to $65 \%$ to $75 \%$ for cases c- 1 to c- 3 , while the reference case with no overhang offers about $55 \%$ likelihoods for such overall views. Probabilities of almost blocked overall views for cases with overhangs increase about $10 \%$ compared to the reference case. Likelihoods of experiencing wide and immersive views reduces about $5 \%$ to $15 \%$ by installing external overhangs to the reference case.

Negative impacts of overhangs on reducing horizontal views are illustrated in Figure 15-2. The likelihoods of poor to almost blocked horizontal FOVs increase to above $25 \%$ to around $40 \%$ in cases c- 1 to c- 3 , which are about $5 \%$ to $20 \%$ higher than the corresponding likelihood of the reference cases, i.e., around $20 \%$. Likelihoods of experiencing wide and immersive horizontal views reduce about $5 \%$ to $20 \%$ for cases c -1 to c-3 compared to the reference case.

Considering probabilities of vertical FOVs in Figure 15-3, cases c-1 to c-3 provide occupants with nearly $80 \%$ likelihoods of poor to almost blocked vertical views while the reference case enables around $70 \%$ likelihoods of such vertical views. Likelihoods of experiencing almost blocked views are increased to around $40 \%$ to $45 \%$ by adding overhangs which are about $5 \%$ to $10 \%$ higher than the corresponding value in the reference case. The occupants of cases c- 1 to c- 3 have around $5 \%$ to $10 \%$ probabilities to experience good to wide and immersive vertical views which is about $5 \%$ to $10 \%$ lower than the corresponding likelihood of the reference cases, i.e., about $15 \%$. 

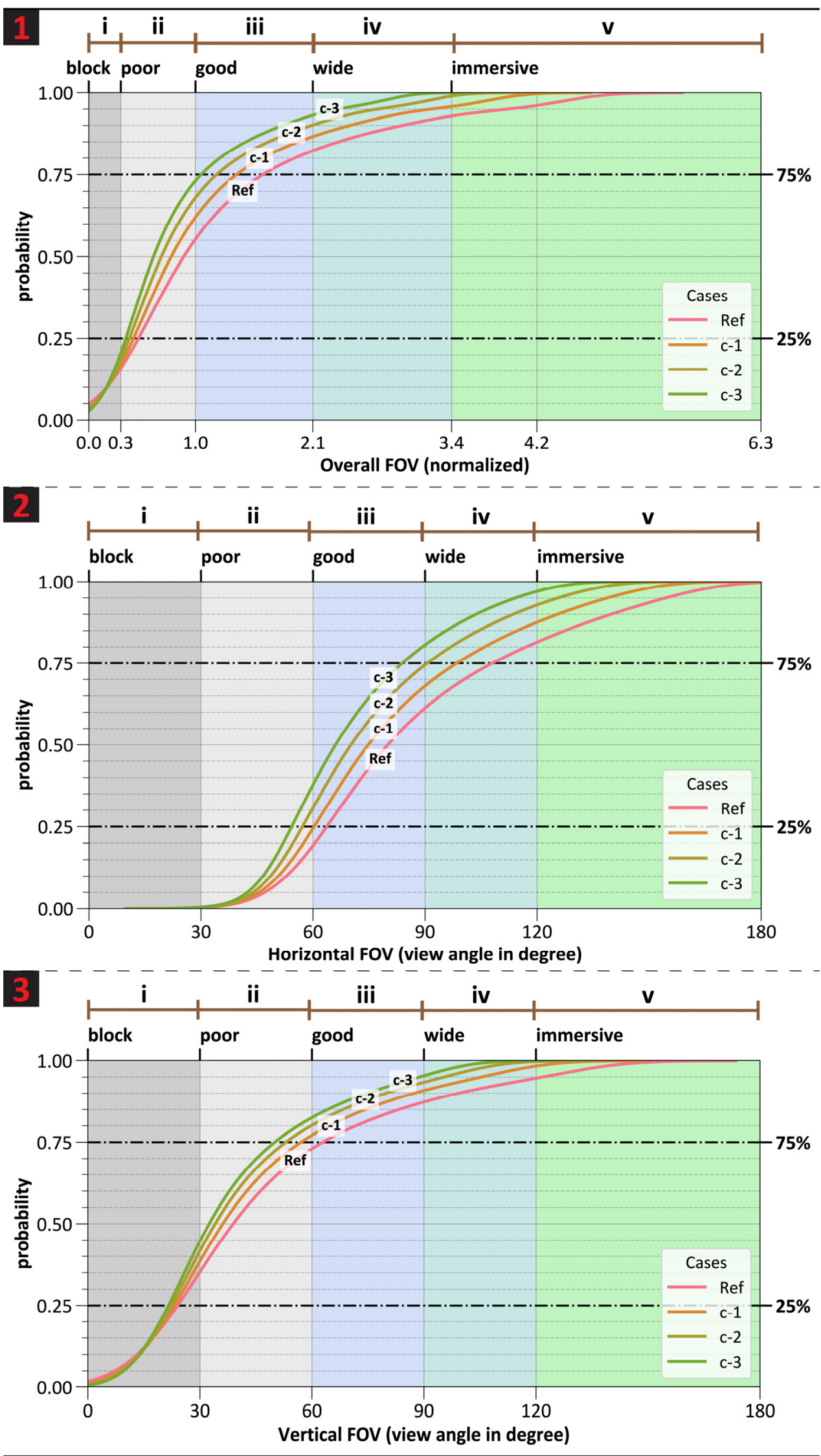

Figure 15. Impacts of external overhangs installed around window edges on the cumulative probabilities of overall, horizontal, and vertical FOVs for case studies of scenario-c. 


\section{Discussion}

The proposed method enables comprehensive visualizations and evaluations of window view access and occupants' visual experience in the pilot study. The false color maps of FOVs in scenario-a visualized the positive effect of increasing window sizes on enabling occupants to experience less blocked and poor views towards more good, wide and immersive views in space. The method showed that the probability densities of experiencing almost blocked and poor views reduced considerably by increasing WWRs to about $40 \%$ and higher, i.e., about 50\%. Increasing WWRs improves likelihoods of experiencing good to wide or immersive views for about $20 \%$. However, the method revealed that WWRs of above $70 \%$ to $100 \%$, i.e., cases a- 7 to a- 9 , offer almost similar visual experiences potentials in space. In other words, increasing WWRs to above 70\% have negligible contributions to the window view access and biophilic visual connections in space. WWRs of $100 \%$ could improve the likelihood of experiencing immersive views by about $5 \%$. Hence, the biophilic aim of immersing occupants to outdoor views and nature could not be met within the boundaries of building geometry. As a promising recommendation, efficient biophilic visual connections, in terms of relatively wide to good views, could be achieved through WWRs of $40 \%$ to $70 \%$. Decreasing WWRs to lower than $40 \%$ could significantly reduce visual connections to outdoors as occupants are exposed to higher probabilities of almost blocked to poor FOVs.

Increasing the window length, i.e., horizontal window frames, offers higher contributions to the overall view access and biophilic visual connections in space compared to the increase in the height, i.e., vertical window frames. Horizontal WWLRs of around $40 \%$ to $80 \%$ could enable efficient biophilic horizontal view access to outdoors. Decreasing WWLRs to less than $40 \%$ could significantly reduce horizontal FOVs. WWLRs of $80 \%$ to $90 \%$ have slight increase in horizontal view angles to outdoors. In terms of the window height, occupants have higher probabilities to experience biophilic vertical FOVs in cases with around $70 \%$ and above WWHRs. Window heights of less than $60 \%$ offer higher probability of poor to almost blocked viewpoints towards outdoors.

The proposed methodological workflow of view access evaluations enables characterizing impacts of multiple windows on the distributions of FOVs in the space. Divided multiple windows uniform the distribution of views in space as suggested by the rendered false color maps and probability patterns of overall and horizontal FOVs. Splitting a single window to small frames, however, could reduce biophilic visual experiences in terms of the probabilities of wide and immersive FOVs.

Adverse impacts of overhangs on individuals' view access are fully characterized in the false color maps, histogram, and cumulative probabilities of overall, horizontal, and vertical FOVs. External overhangs could have from $5 \%$ to above $20 \%$ negative impacts on FOVs in terms of reducing the probabilities of experiencing good, wide, or immersive views as well as increasing the likelihoods of almost blocked and poor views. The negative impacts of external overhangs on FOVs of occupants are higher in standing position compared to seated viewpoints. Increasing the size of external overhangs exacerbates the overall adverse impact on view access to outdoors through the viewpoints in space.

\section{Conclusions}

This paper developed a methodological framework for window view access in architecture based on the typical vision fields of human eyes and biophilia. The method enables comprehensive visualization and probability evaluations of view access potentials in space which could enlighten designers about the impacts of different architectural configurations. The method could potentially promote informative decision-making processes at early stages of architectural design processes, especially in terms of efficient window configurations and indoor-outdoor connections. The pilot study with 15 case studies showed the efficiency of the presented method in spatial visualization and probability evaluations of occupants' visual experiences towards the window and outdoors. Results of the pilot study also confirms that the proposed view access evaluation framework could be developed 
and integrated as a useful and informative module in building simulation tools. The presented window view access methodological framework could also be integrated with the indicators of the view content and qualities and to provide comprehensive evaluations of occupants' visual experiences in architecture. The efficiency and applicability of the proposed method could be further improved by coupling with daylighting modeling tools. Integrated view access and daylighting modeling enables exploring the potential impacts of surface characteristics on visual experiences of occupants in various FOVs. Future research could also study the application and efficiency of the proposed method in complex architectural configurations and geometries.

Overall, the main outlines of the research can be concluded as the following.

- Integrating the typical vision fields of human eyes could constitute a comprehensive fundamental basis to evaluate architectural configurations in terms of overall, horizontal, and vertical FOVs of occupants to outdoors. Evaluations window view access based on typical vision fields enable discussing biophilic visual connections in architecture. The paper, hence, integrated the typical vision with existing recommendations for view ranges and biophilic design to evaluate case studies.

- Using cumulative probabilities of view access enables comparing different architectural configurations by reporting potentials of experiencing different views in space. Cumulative probabilities could show likelihoods of experiencing a specific range of FOV in the space. Cumulative probabilities could also inform potential FOVs which could be experienced from different percentages of viewpoints, e.g., $75 \%$ or $25 \%$, in space.

- Considering the pilot study, the method suggested that increasing the window sizes to $40 \%$ WWRs could significantly reduce almost blocked and poor views in the space, about $25 \%$ to $50 \%$ reductions. WWRs of $40 \%$ and above increase the likelihood of experiencing good to wide FOVs, i.e., from $30 \%$ to $60 \%$. However, increasing WWRs to above $70 \%$ offer slight positive effects, i.e., less than $5 \%$ to $10 \%$, on improving view access potentials, especially in terms of experiencing immersive and wide views. Providing the majority of occupants $(75 \%)$ with immersive views of outdoors, i.e., the ultimate goal of biophilia, could not be addressed through the simulated architectural configurations. WWRs of between $40 \%$ and $70 \%$ could offer optimum view access distributions by offering $75 \%$ likelihoods of experiencing good to wide FOVs and less than $25 \%$ probabilities of poor and blocked views in the cases studies.

- Dividing a single window into multiple smaller frames could uniform the distributions of view access potentials in space by $10 \%$ to $20 \%$ in likelihoods of horizontal FOVs studied for cases with fixed window heights. However, divided multiple windows, especially for very small frames, could have considerable adverse impacts on biophilic visual connections in space by reducing the probability of experiencing higher FOVs such as wide and immersive views.

- External overhangs could have negative impacts on view access to outdoors by about $5 \%$ to $10 \%$, depending on the size of projections.

Author Contributions: Conceptualization, methodology, software, visualization, and writingoriginal draft preparation, M.P.; validation, M.P. and C.M.H.D.; writing—review and editing, All authors; supervision, C.M.H.D., A.P., M.H. and J.-F.L. All authors have read and agreed to the published version of the manuscript.

Funding: This research was funded by the Sentinel North program of Université Laval, made possible, in part, thanks to funding from the Canada First Research Excellence Fund. The APC was also funded by the Sentinel North program.

Institutional Review Board Statement: Not applicable.

Informed Consent Statement: Not applicable.

Conflicts of Interest: The authors declare no conflict of interest. 


\section{Appendix A}
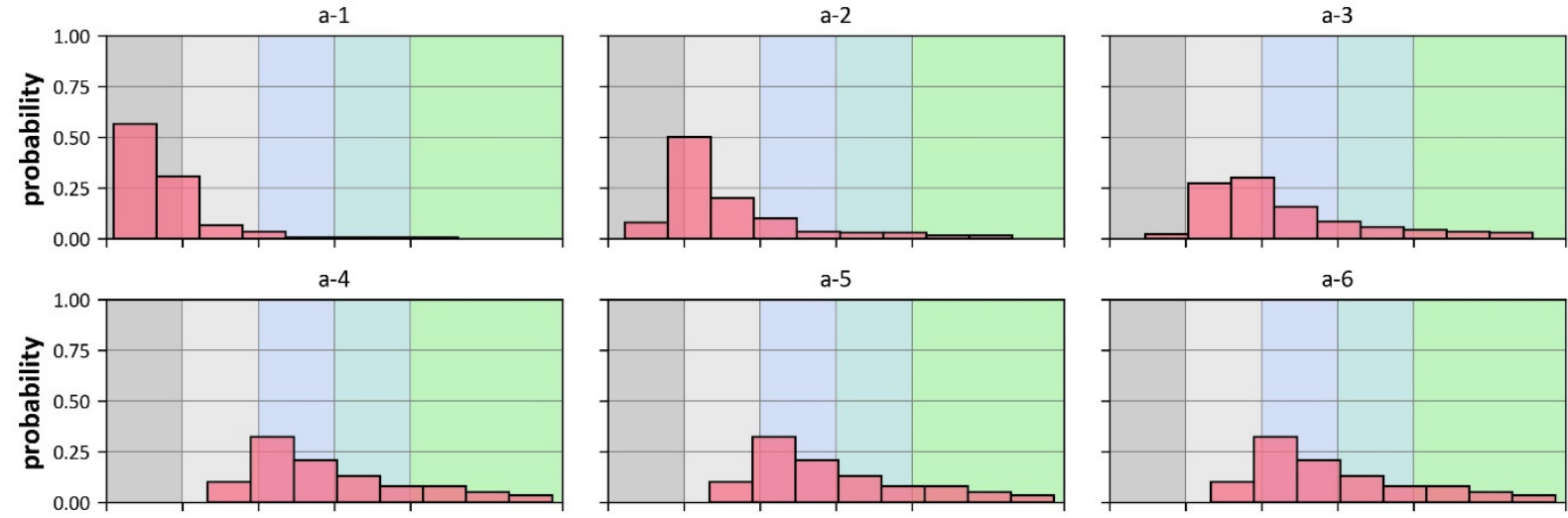

a-6
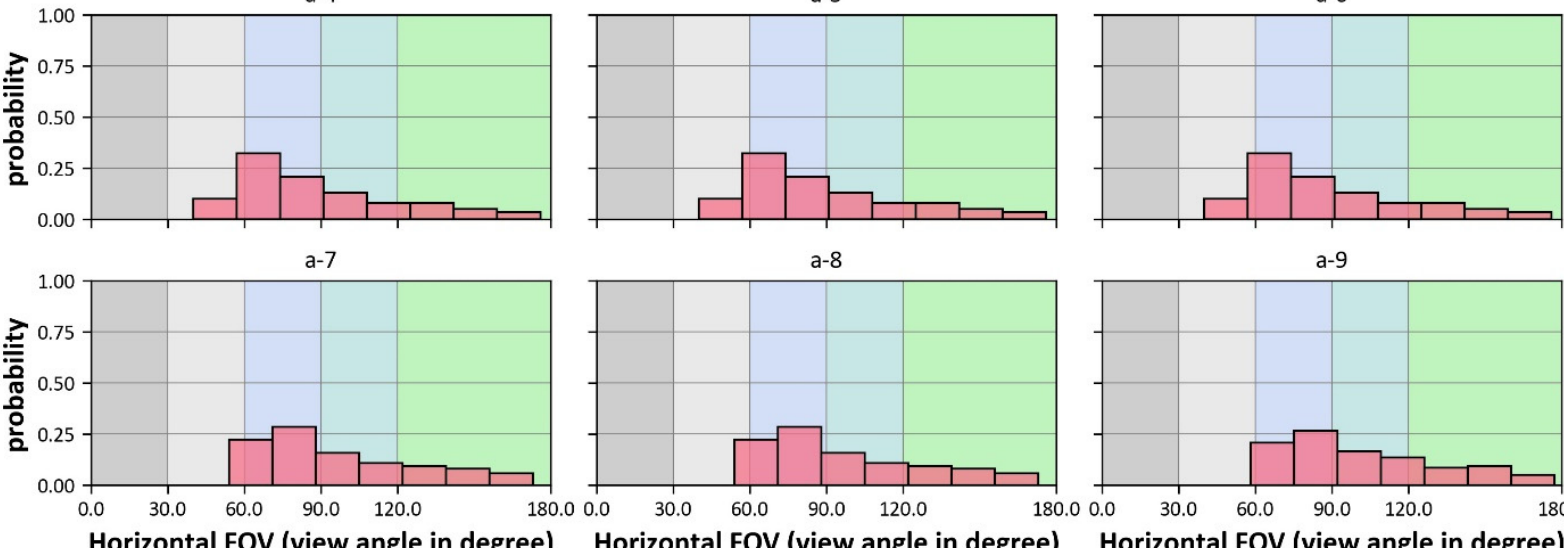

a-

a-9
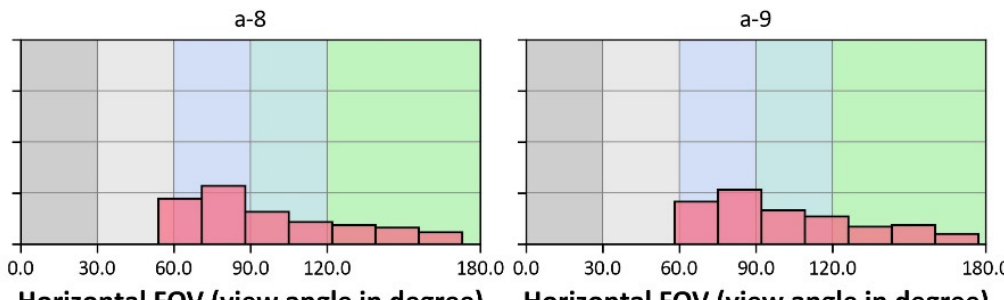

Horizontal FOV (view angle in degree) Horizontal FOV (view angle in degree) Horizontal FOV (view angle in degree)

Figure A1. Histograms of the computed horizontal FOVs for scenario-a, including case studies with different window sizes.
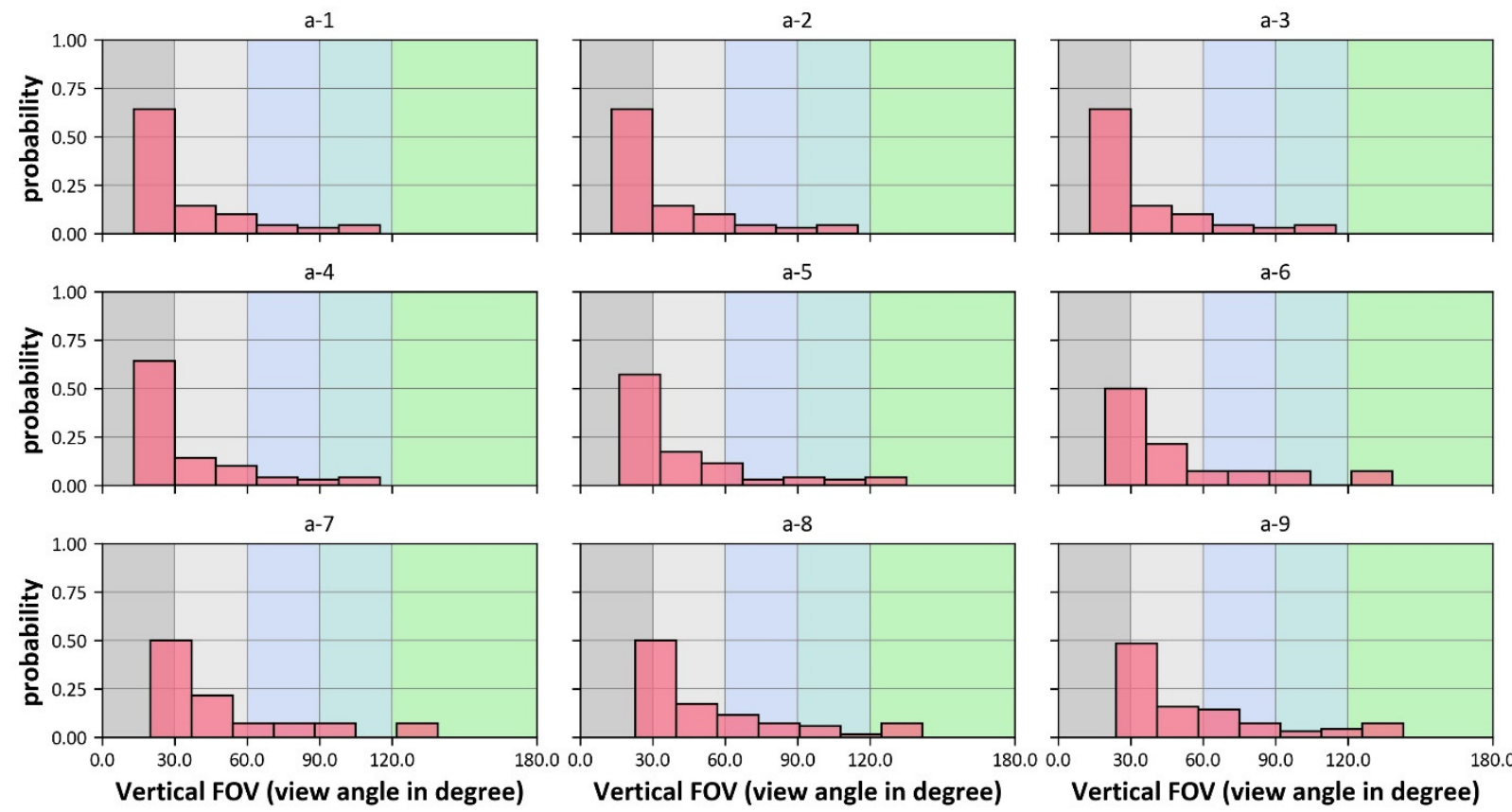

Figure A2. Histograms of the computed vertical FOVs for scenario-a, including case studies with different window sizes. 

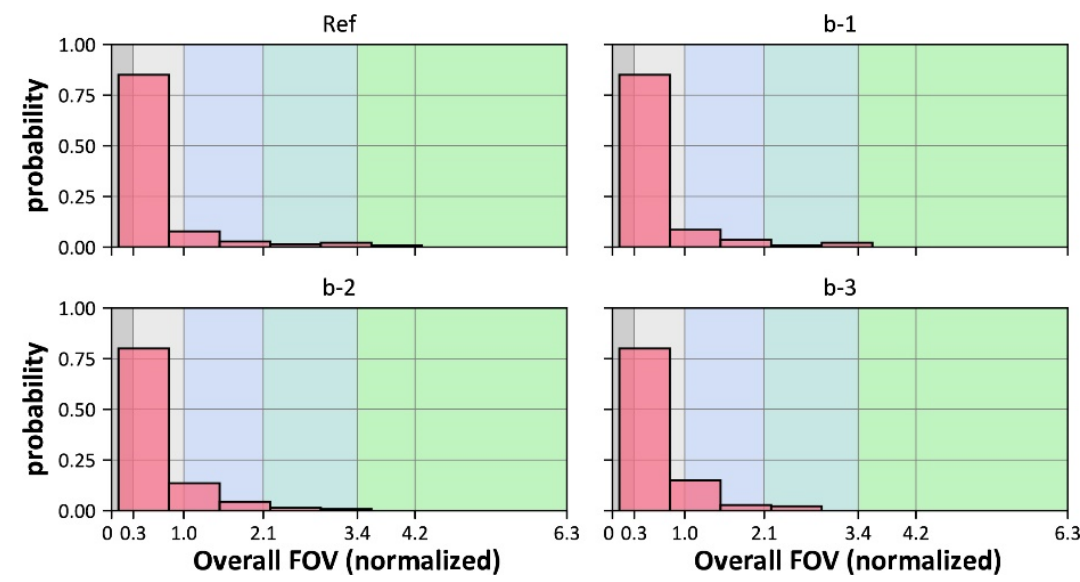

Figure A3. Impacts of dividing to multiple windows on the histograms of the overall FOVs for the case studies of scenario-b.
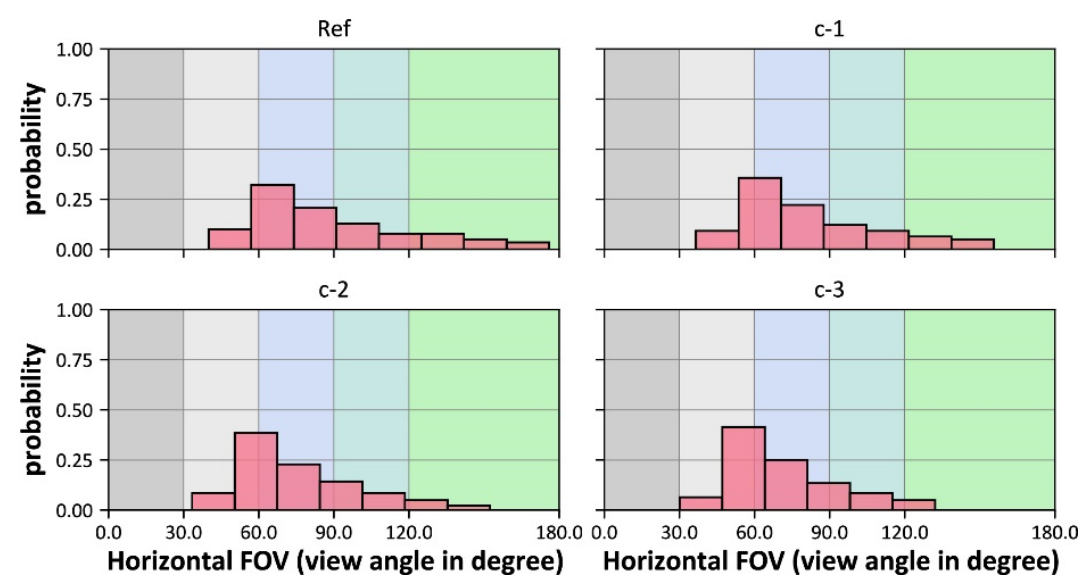

Figure A4. Impacts of external overhangs installed around window edges on the histograms of horizontal FOVs for case studies of scenario-c.
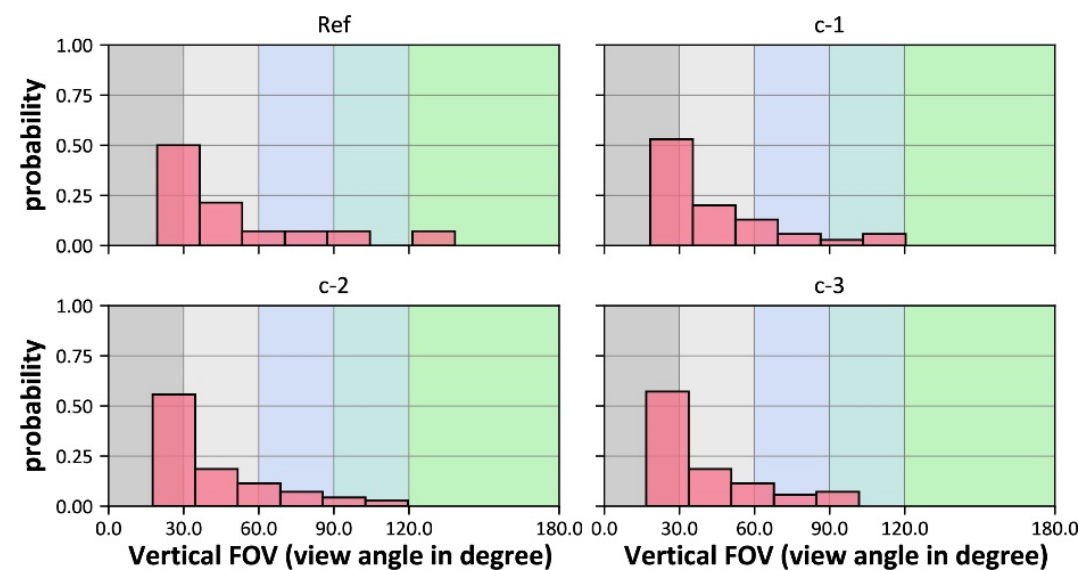

Figure A5. Impacts of external overhangs installed around window edges on the histograms of vertical FOVs for case studies of scenario-c.

\section{References}

1. European Committee For Standardization (CEN). Daylight in Buildings; European Committee For Standardization (CEN): Brussels, Belgium, 2018.

2. Ko, W.H.; Kent, M.; Schiavon, S.; Levitt, B.; Betti, G. A window view quality assessment framework. LEUKOS 2021. [CrossRef]

3. Browning, W.; Ryan, C.; Clancy, J. 14 Patterns of Biophilic Design; Terrapin Bright Green: New York, NY, USA, 2014. 
4. Browning, W.D.; Ryan, C.O. Nature Inside: A Biophilic Design Guide; RIBA Publishing: London, UK, 2020.

5. Kellert, S.R.; Calabrese, E.F. The Practice of Biophilic Design. 2015. Available online: https://www.biophilic-design.com/ (accessed on 15 August 2018).

6. Loftness, V.; Snyder, M. Where Windows Become Doors. In Biophilic Design: The Theory, Science and Practice of Bringing Buildings to Life; Kellert, S.R., Heerwagen, J., Mador, M., Eds.; John Wiley \& Sons: Hoboken, NJ, USA, 2011; pp. 119-131.

7. Heschong, L. Visual Delight in Architecture: Daylight, Vision, and View; Routledge: New York, NY, USA, 2021.

8. McGee, B.; Marshall-Baker, A. Loving nature from the inside out: A Biophilia matrix identification strategy for designers. HERD Health Environ. Res. Des. J. 2015, 8, 115-130. [CrossRef] [PubMed]

9. Ko, W.H.; Schiavon, S.; Zhang, H.; Graham, L.T.; Brager, G.; Mauss, I.; Lin, Y.W. The impact of a view from a window on thermal comfort, emotion, and cognitive performance. Build. Environ. 2020, 175, 106779. [CrossRef]

10. Van den Berg, A.E.; Joye, Y.; Koole, S.L. Why viewing nature is more fascinating and restorative than viewing buildings: A closer look at perceived complexity. Urban For. Urban Green. 2016, 20, 397-401. [CrossRef]

11. Kaplan, R. The role of nature in the context of the workplace. Landsc. Urban Plan. 1993, 26, 193-201. [CrossRef]

12. Cleveland, A.C. Symbiosis Between Biophilic Design and Restorative Healing Environments: The Impact on Overall Well-Being of Urban Dwellers. In Department of Interior Design; Florida State University: Tallahassee, FL, USA, 2014.

13. Aries, M.B.C.; Veitch, J.A.; Newsham, G.R. Windows, view, and office characteristics predict physical and psychological discomfort. J. Environ. Psychol. 2010, 30, 533-541. [CrossRef]

14. Soderlund, J.; Newman, P. Biophilic architecture: A review of the rationale and outcomes. AIMS Environ. Sci. 2015, 2, 950-969.

15. Kellert, S.R. Dimensions, Elements, and Attributes of Biophilic Design. In Biophilic Design: The Theory, Science and Practice of Bringing Buildings to Life; Kellert, S.R., Heerwagen, J., Mador, M., Eds.; John Wiley \& Sons: Hoboken, NJ, USA, 2011 ; pp. 3-19.

16. Gillis, K.; Gatersleben, B. A review of psychological literature on the health and wellbeing benefits of biophilic design. Buildings 2015, 5, 948-963. [CrossRef]

17. van Esch, E.; Minjock, R.; Colarelli, S.M.; Hirsch, S. Office window views: View features trump nature in predicting employee well-being. J. Environ. Psychol. 2019, 64, 56-64. [CrossRef]

18. Lottrup, L.; Stigsdotter, U.K.; Meilby, H.; Claudi, A.G. The Workplace Window View: A Determinant of Office Workers' Work Ability and Job Satisfaction. Landsc. Res. 2013, 40, 57-75. [CrossRef]

19. Sop Shin, W. The influence of forest view through a window on job satisfaction and job stress. Scand. J. For. Res. 2007, 22, 248-253. [CrossRef]

20. Turan, I.; Chegut, A.; Fink, D.; Reinhart, C. Development of view potential metrics and the financial impact of views on office rents. Landsc. Urban Plan. 2021, 215, 104193. [CrossRef]

21. WELL. The WELL Building Standard. Available online: https://standard.wellcertified.com/ (accessed on 20 July 2020).

22. LEED. Daylight and Quality Views, Indoor Environmental Quality in LEED V4. Available online: https://www.usgbc.org/ credits/warehouse-and-distribution-centers-existing-buildings/v4/eq122-0 (accessed on 30 July 2021).

23. International Living Future Institute. Biophilic Design Exploration Guidebook; International Living Future Institute: Seattle, WA, USA, 2017.

24. Heschong Mahone Group. Windows and Offices: A Study of Office Worker Performance and the Indoor Environment; Heschong Mahone Group: Fair Oaks, CA, USA, 2003.

25. Parsaee, M.; Demers, C.M.H.; Hébert, M.; Lalonde, J.F.; Potvin, A. Biophilic, photobiological and energy-efficient design framework of adaptive building façades for Northern Canada. Indoor Built Environ. 2020, 30, 665-691. [CrossRef]

26. Parsaee, M.; Demers, C.M.H.; Hébert, M.; Lalonde, J.F.; Potvin, A. A photobiological approach to biophilic design in extreme climates. Build. Environ. 2019, 154, 211-226. [CrossRef]

27. Parsaee, M.; Demers, C.M.H.; Potvin, A.; Lalonde, J.F.; Inanici, M.N.; Hébert, M. Biophilic photobiological adaptive envelopes for sub-Arctic buildings: Exploring impacts of window sizes and shading panels' color, reflectance, and configuration. Sol. Energy 2021, 220, 802-827. [CrossRef]

28. Ma, J.; Fan, N.; Wang, N. Normal Visual Field. In Advances in Visual Science and Eye Diseases; Springer: Singapore, $2019 ;$ pp. 43-48.

29. Daw, N.W. Visual Development, 3rd ed.; Springer: New York, NY, USA, 2014.

30. Strasburger, H.; Rentschler, I.; Jüttner, M. Peripheral vision and pattern recognition: A review. J. Vis. 2011, 11, 13. [CrossRef]

31. Younis, O.; Al-Nuaimy, W.; Rowe, F.; Alomari, M.H. A Smart Context-Aware Hazard Attention System to Help People with Peripheral Vision Loss. Sensors 2019, 19, 1630. [CrossRef]

32. Strasburger, H. Seven Myths on Crowding and Peripheral Vision. i-Perception 2020, 11, 204166952091305. [CrossRef] [PubMed]

33. Haskins, A.J.; Mentch, J.; Botch, T.L.; Robertson, C. Active vision in immersive, 360 degrees real-world environments. Sci. Rep. 2020, 10, 14304. [CrossRef] [PubMed]

34. Chang, C.; Bang, K.; Wetzstein, G.; Lee, B.; Gao, L. Toward the next-generation VR/AR optics: A review of holographic near-eye displays from a human-centric perspective. Optica 2020, 7, 1563-1578. [CrossRef] [PubMed]

35. Dey, A. Perceptual Characteristics of Visualizations for Occluded Objects in Handheld Augmented Reality. In School of Information Technology \& Mathematical Sciences; University of South Australia: Adelaide, Australia, 2013.

36. Germano, D. Detecting Joint Attention Through Data-Driven Methods; University of Lausanne-HEC Lausanne: Lausanne, Switzerland, 2019. 